BIHEP-TH-2002-21

\title{
Thermodynamics of a deformed Bose gas
}

\author{
Zhe Chang ${ }^{1}$ and Shao-Xia Chen ${ }^{2}$ \\ Institute of High Energy Physics, Chinese Academy of Sciences \\ P.O.Box 918(4), 100039 Beijing, China
}

\begin{abstract}
By making use of the double-time Green function technique, we study thermodynamics of a deformed Bose gas, which describes well properties of density intensive photonic gas and radiation fields of the early universe. General form of statistical distribution function is obtained. We show explicitly the expression of the distribution function in some limitation cases. The free energy, equation of state, specific heat and other thermodynamic properties of the deformed Bose gas are presented.
\end{abstract}

PACS numbers: 05.30.-d, 05.10.-a, 98.80.Cq, 05.70.-a, 51.30.+i

\section{Introduction}

There are two main motivations to study a deformed many-body system. The first one comes from fundamental physics. It is well-known that the noncommutative geometry plays a very important role in unravelling the properties of the Planck scale physics. It has for a long time been suspected that the noncommutative spacetime might be a realistic picture of how spacetime behaves near the Planck scale[1]. In fact, the

\footnotetext{
${ }^{1}$ changz@mail.ihep.ac.cn

${ }^{2}$ ruxanna@mail.ihep.ac.cn
} 
noncommutative geometry naturally enters the theory of open string in a background $B$-field[2]. In particular, the noncommutative geometry makes the holography[3] (e.g. the AdS/CFT correspondence) of a higher dimensional quantum system of gravity and lower dimensional theory possible. A kind of special regularization with exponentially increasing spacetime cutoff has been introduced based on the noncommutative geometry. A very small displacement of the noncommutative deformation parameter from its classical value reduces sharply the entropy of quantum system of gravity. An adequately adopted noncommutative deformation of geometry makes the holography of a higher dimensional quantum system of gravity and lower dimensional theory possible. It was also discovered that simple limits of $M$ theory and superstring theory lead directly to the noncommutative gauge field theory $[4,5]$. The noncommutative field theory has been intensively studied in the past two decads $[6,7]$. The most realistic laboratory for testing the Planck scale physics is the early universe and the black hole physics. From the standard picture of cosmology, the universe started from a very hot, dense phase about 15 billion years ago in the big bang. The very early universe was opaque due to the constant interchange of energy between matter and radiation. Several problems of standard big bang cosmology, such as the horizon, homogeneity, entropy and flatness problems, can be explained under the assumption that the very early universe underwent a period of extremely rapid inflation[8], driven by the potential of some assumed inflation fields. In particular, the inflation scenario is also able to explain the observed perturbations in the cosmic microwave background radiation $[9,10]$, namely as originating ultimately from quantum fluctuations of the inflation field. However, in most of the current models of inflation, the physical length of perturbations was much smaller than the Planck length at the beginning of inflation[11, 12]. It is then of interest to investigate whether the predictions of the spectrum of cosmological perturbations are sensitive to the unknown super-Planck-scale physics[13]-[16]. Recent studies[17]-[24] by physicists and astrophysicists show that without strange inflation fields but just the noncommutative geometry and the deformed radiation dominated universe subject 
to the noncommutative spacetime quantization indeed give rise to inflation as the radiation temperature exceeds the Planck temperature. Based on the noncommutative geometry and the deformed radiation, one can even get an alternative explanation for the horizon, homogeneity, entropy and flatness problems in the standard cosmology.

Another motivation comes from quantum optics as well as molecular and atomic spectroscopy. A fascinating development of quantum optics in last two decades has taken place within two specific areas, one concerning the study of nonclassical states of light, and the other studying the interaction of light with matter. The nonclassical states of light offer the possibility of the so-called reduction of quantum noise below the standard limit, determined by the Heisenberg's uncertainty relation. Study on interaction of light with matter shows that the old Bohr quantum jumps are real. Dynamics of deformed photonic field interacting with matter presented many interesting properties both in the two important areas[25]-[29]. Great success has also been made by making use of a deformed oscillator to study molecular spectrum[30]-[32].

In this paper, we study thermodynamics of a deformed Bose gas, and in particular, of a deformed radiation field. On the topic, many papers have been published in more than ten years[33]-[46]. However, we still have not a general form of statistical distribution function of the deformed Bose gas, which should reduce to the Bose-Einstein distribution function for an ideal Bose gas in the $q=1$ limit. In this situation, it is difficult to investigate systematically the influence of the deformation on thermodynamic characteristics of a Bose system, which is interesting in fundamental physics and other subjects as discussed above. For this purpose, by making use of the double-time Green function technique, we obtain an algebra equation, which should be satisfied by the distribution function of a deformed Bose gas. The equation for distribution function can not be solved exactly in general. The situation is similar to the case of magnetic lattices. From this point of view, a deformed Bose gas is really a strongly correlated system. In the case of $q \rightarrow 1$, we give a useful expression of the distribution function for a deformed Bose gas. Thermodynamic properties, such as internal energy, specific 
heat, free energy and equation of state, are discussed in detail.

The paper is organized as follows. In section 2, a deformed Bose gas is discussed. We get the distribution function for a deformed Bose gas by using the double-time Green function technique in section 3. In section 4, we calculate some thermodynamic quantities for a deformed photon gas and compare them with those for the ideal Bose gas. Concluding remarks are given in section 5 .

\section{A deformed Bose gas system}

The Lagrange density of a scalar field $\phi(x)$ with mass $m$ reads

$$
\mathcal{L}(x)=\frac{\hbar^{2}}{2} \frac{\partial \phi}{\partial x_{\mu}} \frac{\partial \phi}{\partial x^{\mu}}-\frac{1}{2} m^{2} c^{2} \phi^{2} .
$$

The Euler-Lagrange equation immediately leads to the Klein-Gordon equation

$$
\left(\hbar^{2} \frac{\partial}{\partial x_{\mu}} \frac{\partial}{\partial x^{\mu}}+m^{2} c^{2}\right) \phi(x)=0 .
$$

The canonically conjugate field is

$$
\pi(x)=\frac{\partial \mathcal{L}}{\partial \dot{\phi}(x)},
$$

which leads to the Hamilton density

$$
\mathcal{H}(x)=\frac{1}{2}\left(\frac{1}{\hbar^{2}} \pi^{2}(x)+(\hbar \nabla \phi(x))^{2}+m^{2} c^{2} \phi^{2}(x)\right) .
$$

The Fourier decomposition of the scalar field is of the form,

$$
\begin{aligned}
& \phi(x)=\int \frac{d^{3} k c}{\sqrt{(2 \pi)^{3} 2 \hbar \omega_{k}}}\left(a(k) e^{-i k x}+a^{\dagger}(k) e^{i k x}\right), \\
& \pi(x)=\int \frac{d^{3} k i \hbar^{2} \omega_{k}}{\sqrt{(2 \pi)^{3} 2 \hbar \omega_{k}}}\left(-a(k) e^{-i k x}+a^{\dagger}(k) e^{i k x}\right),
\end{aligned}
$$

where we have used the notation $\omega_{k}=\frac{c}{\hbar} \sqrt{(\hbar \mathbf{k})^{2}+m^{2} c^{2}}$. The canonical quantization of the scalar field reads

$$
[\hat{\phi}(\mathbf{x}, t), \hat{\pi}(\mathbf{y}, t)]=i \hbar c \delta^{3}(\mathbf{x}-\mathbf{y})
$$


In terms of the Fourier modes $\hat{a}(k)$ and $\hat{a}^{\dagger}(k)$, the commutation relations can be rewritten into the form

$$
\left[\hat{a}(k), \hat{a}^{\dagger}\left(k^{\prime}\right)\right]=\delta^{3}\left(\mathbf{k}-\mathbf{k}^{\prime}\right)
$$

It is not difficult to express the Hamiltonian in terms of these Fourier modes,

$$
\hat{H}=\frac{1}{2} \int d^{3} k \hbar \omega_{k}\left(\hat{a}(k) \hat{a}^{\dagger}(k)+\hat{a}^{\dagger}(k) \hat{a}(k)\right)
$$

In the quantum field theory, the vacuum state is defined as follows

$$
\hat{a}(k)|0\rangle=0
$$

And the $N$-particle Fock space can be written down directly as

$$
\left|n\left(k_{1}\right), n\left(k_{2}\right), \cdots, n\left(k_{m}\right)\right\rangle=\frac{\left(\hat{a}^{\dagger}\left(k_{1}\right)\right)^{n\left(k_{1}\right)}}{\sqrt{n\left(k_{1}\right) !}} \frac{\left(\hat{a}^{\dagger}\left(k_{2}\right)\right)^{n\left(k_{2}\right)}}{\sqrt{n\left(k_{2}\right) !}} \cdots \frac{\left(\hat{a}^{\dagger}\left(k_{m}\right)\right)^{n\left(k_{m}\right)}}{\sqrt{n\left(k_{m}\right) !}}|0\rangle
$$

To measure how many particles of a certain momentum exist in the field system, one can introduce the particle number operator $\hat{N}(k)$,

$$
\hat{N}(k)=\hat{a}^{\dagger}(k) \hat{a}(k)
$$

It is easy to check that

$$
\hat{N}\left(k_{i}\right)\left|n\left(k_{1}\right), n\left(k_{2}\right), \cdots, n\left(k_{m}\right)\right\rangle=n\left(k_{i}\right)\left|n\left(k_{1}\right), n\left(k_{2}\right), \cdots, n\left(k_{m}\right)\right\rangle
$$

Thus, a field can be seen as a many-body system that consists of free harmonic oscillators with different momentum. Properties of the field system can be described exactly by the Weyl-Heisenberg algebra,

$$
\begin{aligned}
& {\left[\hat{a}, \hat{a}^{\dagger}\right]=1,} \\
& {[\hat{N}, \hat{a}]=-\hat{a}, \quad\left[\hat{N}, \hat{a}^{\dagger}\right]=\hat{a}^{\dagger},}
\end{aligned}
$$

with the Fock space $|n\rangle=\frac{\left(\hat{a}^{\dagger}\right)^{n}}{\sqrt{n !}}|0\rangle$ as its representation. All properties, in particular, the thermodynamic properties of the many-body system can be investigated systematically, by making use of the Hamiltonian (8) and the Weyl-Heisenberg algebra (13). 
The natural quantum of the quantum field theory on noncommutative geometry is the so-called deformed harmonic oscillator. On the topic of deformed oscillators, a lot of papers have been published[47]-[49]. Similar to the case of an ordinary quantum field system, the many-body properties of a noncommutative quantum field system can be described by a collection of the deformed harmonic oscillators,

$$
\hat{H}_{q}=\frac{1}{2} \sum_{\mathbf{k}} \hbar \omega_{k}\left(\hat{a}_{q}(k) \hat{a}_{q}^{\dagger}(k)+\hat{a}_{q}^{\dagger}(k) \hat{a}_{q}(k)\right) .
$$

The deformed harmonic oscillator is related with the simple harmonic oscillator as follows[50],

$$
\hat{a}_{q}=\sqrt{\frac{[\hat{N}]_{q}}{\hat{N}}} \hat{a}, \quad \hat{a}_{q}^{\dagger}=\hat{a}^{\dagger} \sqrt{\frac{[\hat{N}]_{q}}{\hat{N}}},
$$

where we have used the notation $[x]_{q}=\frac{q^{x}-q^{-x}}{q-q^{-1}}$.

The deformed Weyl-Heisenberg algebra reads

$$
\begin{aligned}
& \hat{a}_{q} \hat{a}_{q}^{\dagger}-q \hat{a}_{q}^{\dagger} \hat{a}_{q}=q^{-\hat{N}}, \\
& {\left[\hat{N}, \hat{a}_{q}^{\dagger}\right]=\hat{a}_{q}^{\dagger}, \quad\left[\hat{N}, \hat{a}_{q}\right]=-\hat{a}_{q} .}
\end{aligned}
$$

The representation of the deformed Weyl-Heisenberg algebra is obtained by constructing the Fock space,

$$
\begin{aligned}
& |n\rangle_{q}=\frac{\left(\hat{a}_{q}^{\dagger}\right)^{n}}{\sqrt{[n]_{q} !}}|0\rangle, \\
& \hat{a}_{q}|n\rangle_{q}=\sqrt{[n]_{q}}|n-1\rangle_{q}, \quad \hat{a}_{q}^{\dagger}|n\rangle_{q}=\sqrt{[n+1]_{q}}|n+1\rangle_{q},
\end{aligned}
$$

where $[n]_{q} ! \equiv[n]_{q}[n-1]_{q} \cdots[2]_{q}[1]_{q}$. It is easy to check that one still can measure how many particles of a certain momentum exist in the deformed field system by making use of the particle number operator $\hat{N}(k)$,

$$
\hat{N}\left(k_{i}\right)\left|n\left(k_{1}\right), n\left(k_{2}\right), \cdots, n\left(k_{m}\right)\right\rangle_{q}=n\left(k_{i}\right)\left|n\left(k_{1}\right), n\left(k_{2}\right), \cdots, n\left(k_{m}\right)\right\rangle_{q}
$$

The Hamiltonian of a deformed harmonic oscillator[51]-[54] can be written as

$$
\hat{H}_{q}=\frac{\hbar \omega}{2}\left([\hat{N}]_{q}+[\hat{N}+1]_{q}\right)
$$

Below we will calculate the distribution function of a deformed harmonic oscillator system by making use of the double-time Green function technique. 


\section{Distribution function}

The double-time Green function (retarded or advanced) for a Bose system[55, 56] is generally defined as

$$
G_{\rho}\left(t-t^{\prime}\right)=-\frac{i}{2}\left((\rho+1) \theta\left(t-t^{\prime}\right)+(\rho-1) \theta\left(t^{\prime}-t\right)\right)\left\langle\left[\hat{A}(t), \hat{B}\left(t^{\prime}\right)\right]\right\rangle
$$

where we have used the notations,

$$
\begin{aligned}
& \hat{A}(t) \equiv \exp \left(\frac{i H t}{\hbar}\right) \hat{A} \exp \left(\frac{-i H t}{\hbar}\right), \\
& \langle\hat{O}\rangle=Z^{-1} \operatorname{Tr}\left(e^{-\beta H} \hat{O}\right), \quad Z=\operatorname{Tr}\left(e^{-\beta H}\right),
\end{aligned}
$$

here $\rho=1$ for the retarded one and $\rho=-1$ for the advanced one.

In Eq.(20), setting

$$
\hat{A}(t) \equiv \hat{a}(t), \quad \hat{B}\left(t^{\prime}\right) \equiv \hat{a}^{\dagger}\left(t^{\prime}\right), \quad \rho=1,
$$

(the retarded Green function is assumed), we get

$$
G_{R}\left(t-t^{\prime}\right)=-i \theta\left(t-t^{\prime}\right)\left\langle\hat{a}(t) \hat{a}^{\dagger}\left(t^{\prime}\right)-\hat{a}^{\dagger}\left(t^{\prime}\right) \hat{a}(t)\right\rangle
$$

The equation of motion of the retarded Green function reads

$i \hbar \frac{\partial}{\partial t} G_{R}\left(t-t^{\prime}\right)=\hbar\left(\delta\left(t-t^{\prime}\right)\left\langle\hat{a}(t) \hat{a}^{\dagger}\left(t^{\prime}\right)-\hat{a}^{\dagger}\left(t^{\prime}\right) \hat{a}(t)\right\rangle+\theta\left(t-t^{\prime}\right)\left\langle\frac{d \hat{a}(t)}{d t} \hat{a}^{\dagger}\left(t^{\prime}\right)-\hat{a}^{\dagger}\left(t^{\prime}\right) \frac{d \hat{a}(t)}{d t}\right\rangle\right)$.

The Heisenberg equation for the operator $\hat{a}(t)$

$$
i \hbar \frac{d \hat{a}(t)}{d t}=[\hat{a}(t), \hat{H}(t)]
$$

gives,

$$
\frac{d \hat{a}(t)}{d t}=-i \frac{\omega}{2}\left(q^{\hat{N}+1}+q^{-(\hat{N}+1)}\right) \hat{a}(t)
$$

Then, we have

$$
i \hbar \frac{\partial}{\partial t} G_{R}\left(t-t^{\prime}\right)=\hbar \delta\left(t-t^{\prime}\right)+\frac{\hbar \omega}{2}\left(q^{\langle\hat{N}+1\rangle}+q^{-\langle\hat{N}+1\rangle}\right) G_{R}\left(t-t^{\prime}\right)
$$


Making use of the Fourier transformation

$$
G_{R}\left(t-t^{\prime}\right)=\int_{-\infty}^{\infty} \frac{d E}{2 \pi \hbar} G_{R}(E) e^{-i E\left(t-t^{\prime}\right) / \hbar}
$$

we get

$$
G_{R}(E)=\frac{\hbar}{E-\frac{\hbar \omega\left(q^{\langle\hat{N}+1\rangle}+q^{-\langle\hat{N}+1\rangle}\right)}{2}} .
$$

The spectrum theorem of Green functions tells us

$$
\left\langle\hat{a}^{\dagger}\left(t^{\prime}\right) \hat{a}(t)\right\rangle=i \int_{-\infty}^{\infty} d E \frac{\left(G_{R}\left(E+i 0^{\dagger}\right)-G_{R}\left(E-i 0^{\dagger}\right)\right) e^{-i E\left(t-t^{\prime}\right) / \hbar}}{2 \pi \hbar\left(e^{\beta E}-1\right)} .
$$

Then, we can write formally the distribution function of a deformed Bose gas as

$$
n(\varepsilon)=\langle\hat{N}\rangle=\frac{1}{\exp \left(\frac{\hbar \omega \beta\left(q^{\langle\hat{N}+1\rangle}+q^{-\langle\hat{N}+1\rangle}\right)}{2}\right)-1} .
$$

Evidently, in the case of $q \rightarrow 1, n(\varepsilon)$ recovers the familiar Bose-Einstein distribution function. In principle, all interesting properties of a deformed Bose gas can be obtained by solving this equation for distribution function. This is to be one of the most advantageous features of the Green function approach.

Now we try to solve Eq.(28) to gain an insight into the influence of the deformation on the distribution function and to compare the deformed distribution function with the Bose-Einstein distribution function.

Expanding the right hand side of Eq.(28) in the neighborhood of $q=1$ to the second order, we get an exactly solvable equation for the deformed distribution function. The solution is of the form,

$$
\begin{aligned}
n(\varepsilon)= & -\frac{1}{\delta^{2} \beta \varepsilon e^{\beta \varepsilon}}\left(\delta^{2} \beta \varepsilon e^{\beta \varepsilon}+\left(e^{\beta \varepsilon}-1\right)^{2}\right) \\
& +\frac{1}{\delta^{2} \beta \varepsilon e^{\beta \varepsilon}} \sqrt{\left(\delta^{2} \beta \varepsilon e^{\beta \varepsilon}+\left(e^{\beta \varepsilon}-1\right)^{2}\right)^{2}-\delta^{2} \beta \varepsilon e^{\beta \varepsilon}\left(\delta^{2} \beta \varepsilon e^{\beta \varepsilon}-2\left(e^{\beta \varepsilon}-1\right)\right)},
\end{aligned}
$$

where we have used the notation $\delta \equiv q-1$ for short.

We note that it is difficult to compare the distribution function of the deformed Bose gas, Eq.(29), with the Bose-Einstein distribution function directly. For the aim, 
we rewrite Eq.(29) as follows,

$$
n(\varepsilon)=\frac{\left(e^{\beta \varepsilon}-1\right)-2^{-1} \delta^{2} \beta \varepsilon e^{\beta \varepsilon}}{\left(e^{\beta \varepsilon}-1\right)^{2}+\delta^{2} \beta \varepsilon e^{\beta \varepsilon}}-\frac{\delta^{2} \beta \varepsilon e^{\beta \varepsilon}\left(\delta^{2} \beta \varepsilon e^{\beta \varepsilon}-2\left(e^{\beta \varepsilon}-1\right)\right)^{2}}{\left(2 \delta^{2} \beta \varepsilon e^{\beta \varepsilon}+2\left(e^{\beta \varepsilon}-1\right)^{2}\right)^{3}} .
$$

One can further expand the distribution function into a series over deformation parameter $\delta$,

$$
n(\varepsilon)=\frac{1}{e^{\beta \varepsilon}-1}-\delta^{2}\left(\frac{\beta \varepsilon e^{\beta \varepsilon}}{2\left(e^{\beta \varepsilon}-1\right)^{2}}+\frac{\beta \varepsilon e^{\beta \varepsilon}}{\left(e^{\beta \varepsilon}-1\right)^{3}}+\frac{\beta \varepsilon e^{\beta \varepsilon}}{2\left(e^{\beta \varepsilon}-1\right)^{4}}\right) .
$$

From the above form of the distribution function of the deformed Bose gas, we see clearly that, at the case of $q=1$, the Bose-Einstein distribution is recovered. To further investigate the effect of deformation on the distribution function, we present a plot of the distribution function in Fig.1.

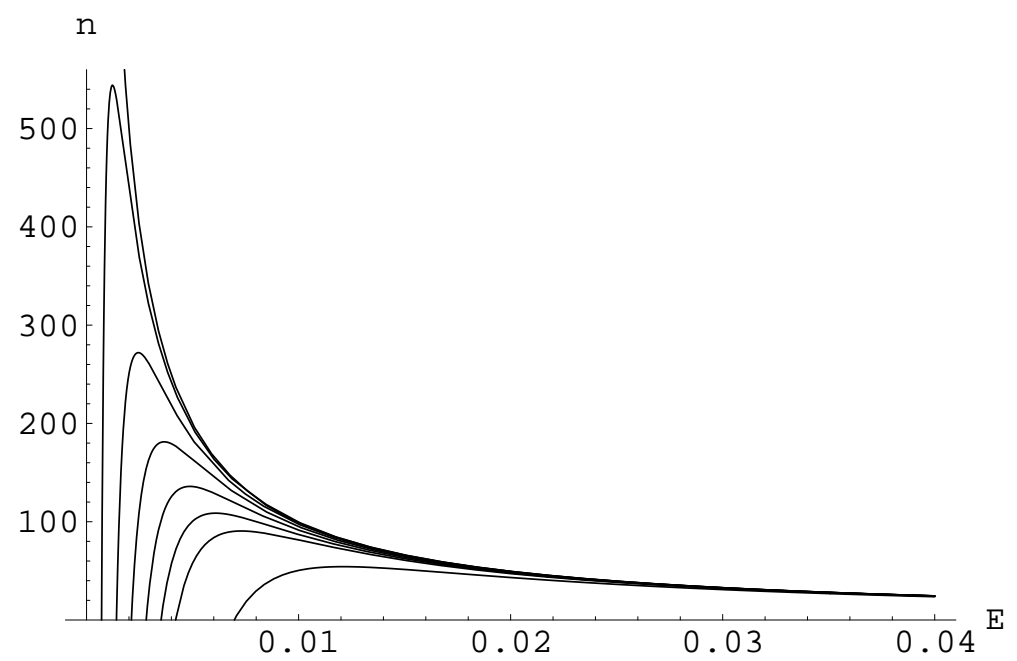

FIG.1 The distribution function of a deformed Bose gas

( start from up $q=1.000,1.001,1.002,1.003,1.004,1.005,1.006,1.010$, here we have used the notation $\beta \varepsilon=E$.)

To describe behaviors of the distribution function of the deformed Bose gas near the ground state more accurately, we give another plot of the deformed distribution function in Fig.2. 


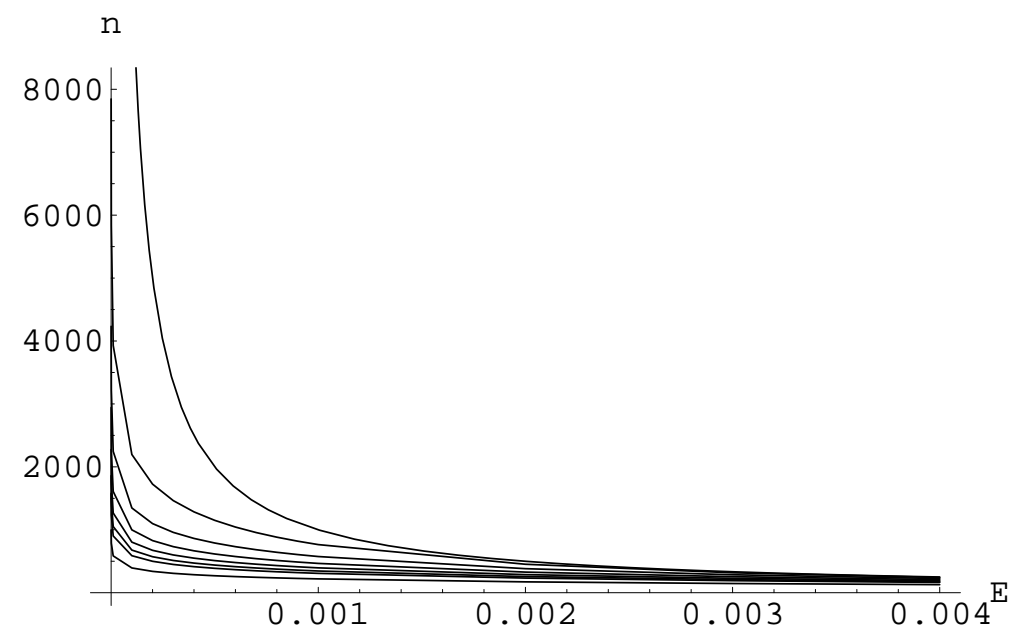

FIG. 2 The behaviors of distribution function of a deformed Bose gas near the ground state

(start from up $q=1.000,1.001,1.002,1.003,1.004,1.005,1.006,1.010$, here we have used the notation $\beta \varepsilon=E$.)

\section{Thermodynamic properties}

In this section, we concentrate on the study of thermodynamic properties of a deformed photonic field. As being well-known, the dispersion relation of a photon is

$$
\epsilon=c p
$$

We know that a photonic field can be described by a collection of simple harmonic oscillators and have relation

$$
\hat{H}=\hbar \omega\left(\hat{N}+\frac{1}{2}\right)
$$

One can then read out that

$$
p=\frac{\hbar \omega}{c}\left(n+\frac{1}{2}\right) .
$$

This relation and the Hamiltonian (19) give the dispersion relation of a deformed Bose gas,

$$
\varepsilon=\frac{1}{2} \hbar \omega\left(\left[\frac{c}{\hbar \omega} p+\frac{1}{2}\right]_{q}+\left[\frac{c}{\hbar \omega} p-\frac{1}{2}\right]_{q}\right) .
$$


Expanding the right hand side of (35) in the neighborhood of $q=1$ to the second order, we get

$$
\varepsilon=c p-\frac{\delta^{2}}{24} c p+\frac{\delta^{2}}{6 \hbar^{2} \omega^{2}} c^{3} p^{3} .
$$

The density of energy levels is as follows,

$$
\begin{aligned}
a(\varepsilon)= & \frac{8 \pi V}{3 h^{3} c^{3}} \cdot \frac{3 \sqrt{3 B} \varepsilon+\sqrt{4 A^{3}+27 B \varepsilon^{2}}}{4 B \sqrt{4 A^{3}+27 B \varepsilon^{2}}} \cdot \frac{\left(-2 \times 6^{1 / 3} A+\left(18 \sqrt{B} \varepsilon+2 \sqrt{3\left(4 A^{3}+27 B \varepsilon^{2}\right)}\right)^{2 / 3}\right)^{2}}{\left(18 \sqrt{B} \varepsilon+2 \sqrt{3\left(4 A^{3}+27 B \varepsilon^{2}\right)}\right)^{1 / 3}} \\
& \times \frac{9 \sqrt{B} \varepsilon+\sqrt{3\left(4 A^{3}+27 B \varepsilon^{2}\right)}+6^{1 / 3} A\left(18 \sqrt{B} \varepsilon+2 \sqrt{3\left(4 A^{3}+27 B \varepsilon^{2}\right)}\right)^{1 / 3}}{\left(9 \sqrt{B} \varepsilon+\sqrt{3\left(4 A^{3}+27 B \varepsilon^{2}\right)}\right)^{2}},
\end{aligned}
$$

where we have used the notations $A \equiv\left(1-\frac{\delta^{2}}{24}\right) c$ and $B \equiv \frac{\delta^{2}}{6 \hbar^{2} \omega^{2}} c^{3}$.

The mean value of the total energy of the system has the form,

$$
\bar{E}=\int_{0}^{\infty} \varepsilon n(\varepsilon) a(\varepsilon) d \varepsilon
$$

To calculate $\bar{E}$, we expand the right hand side of (37) to the third order in the neighborhood of $q=1$ and rewrite the density of energy levels as the form

$$
a(\varepsilon)=\frac{8 \pi V}{3 h^{3} c^{3}}\left(3-\frac{17}{8} \delta^{2}\right) \varepsilon^{2} .
$$

Then we get

$$
\bar{E}=\frac{8 \pi^{5} V}{15} \frac{(k T)^{4}}{(h c)^{3}}-\pi V \delta^{2}\left(\frac{8 \pi^{2}}{3}+\frac{11 \pi^{4}}{15}+48 \zeta(3)\right) \frac{(k T)^{4}}{(h c)^{3}},
$$

where $\zeta(n)$ is the Riemann zeta function.

We can further calculate the grand partition function $\ln \Xi$ through the thermodynamic relation $\bar{E}=-\frac{\partial}{\partial \beta} \ln \Xi$,

$$
\ln \Xi=\frac{8 \pi^{5} V}{45 h^{3} c^{3}}(k T)^{3}-\frac{\pi V \delta^{2}}{h^{3} c^{3}}\left(\frac{8 \pi^{2}}{9}+\frac{11 \pi^{4}}{45}+16 \zeta(3)\right)(k T)^{3} .
$$

It is a straightforward calculation to obtain thermodynamic functions by making use of the grand partition function. The pressure of the deformed Bose gas is

$$
P=\frac{1}{\beta} \frac{\partial}{\partial V} \ln \Xi=\frac{8 \pi^{5}}{45 h^{3} c^{3}}(k T)^{4}-\frac{\pi \delta^{2}}{h^{3} c^{3}}\left(\frac{8 \pi^{2}}{9}+\frac{11 \pi^{4}}{45}+16 \zeta(3)\right)(k T)^{4} .
$$


The free energy of the deformed Bose gas is of the form,

$$
F=-\frac{8 \pi^{5} V}{45} \frac{(k T)^{4}}{(h c)^{3}}+\pi V \delta^{2}\left(\frac{8 \pi^{2}}{9}+\frac{11 \pi^{4}}{45}+16 \zeta(3)\right) \frac{(k T)^{4}}{(h c)^{3}} .
$$

The entropy of the deformed Bose gas is as

$$
S=\frac{U-F}{T}=\frac{32 \pi^{5}}{45} \frac{k^{4} T^{3}}{h^{3} c^{3}} V-\pi \delta^{2}\left(\frac{32 \pi^{2}}{9}+\frac{44 \pi^{4}}{45}+64 \zeta(3)\right) \frac{k^{4} T^{3}}{h^{3} c^{3}} V .
$$

The first term in the above equation is just the entropy of an ordinary Bose gas. And the terms following it denote the effect of noncommutative deformation of geometry on the entropy. It is clear that the effect can not be ignored at least in the high temperature region, where the early universe lived. We give a plot of the entropy vs temperature for different deformation parameters in Fig. 3.

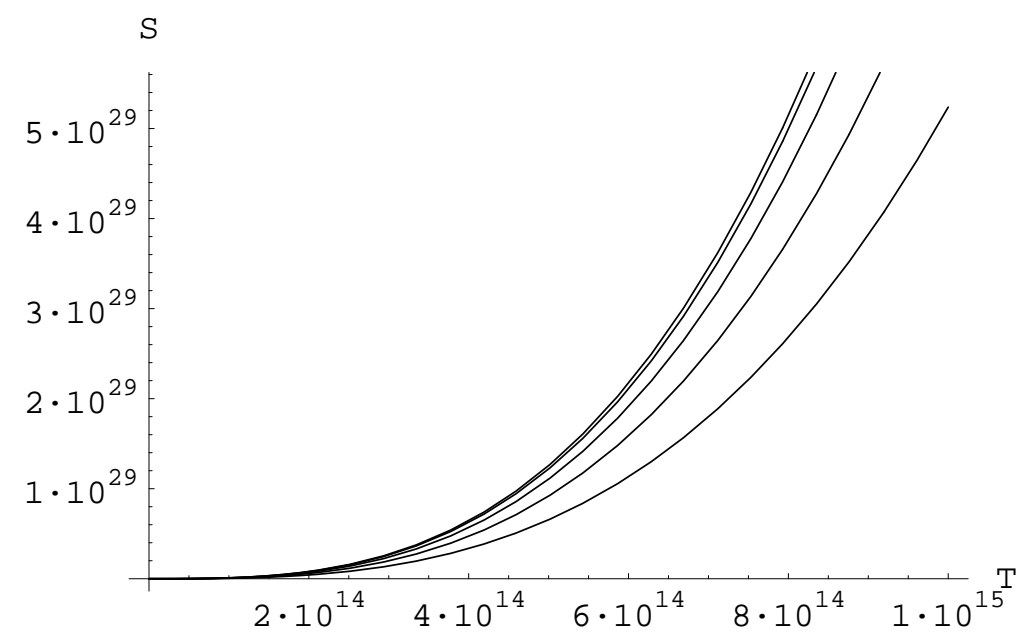

FIG.3 The entropy per unit volume of a deformed Bose gas ( start from up $q=1.0,1.1,1.2,1.3,1.4$ ).

Finally, the specific heat of the deformed Bose gas is

$$
C_{V}=T\left(\frac{\partial S}{\partial T}\right)_{V}=\frac{32 \pi^{5} V}{15} \frac{k^{4} T^{3}}{h^{3} c^{3}}-\pi V \delta^{2}\left(\frac{32 \pi^{2}}{3}+\frac{44 \pi^{4}}{15}+192 \zeta(3)\right) \frac{k^{4} T^{3}}{h^{3} c^{3}}
$$

At the case of $q=1$, we recover the result for an ordinary Bose gas. To show the effect of deformation on the specific heat, we give a plot of the specific heat per unit volume of a deformed Bose gas vs temperature for different deformation parameters in Fig. 4.

From the above discusses, we can also obtain the Planck's formula for the deformed Bose gas, 


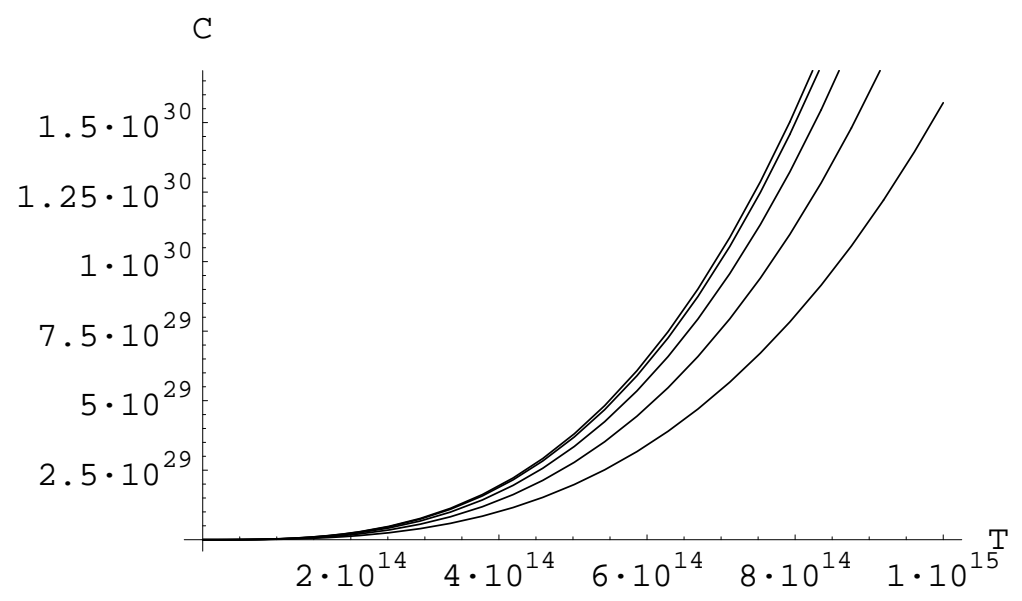

FIG.4 The specific heat per unit volume of a deformed Bose gas

$$
\begin{aligned}
(\text { start from up } q & =1.0,1.1,1.2,1.3,1.4) . \\
u(\omega, T)= & \frac{\hbar \omega^{3}}{c^{3} \pi^{2}\left(e^{\beta \hbar \omega}-1\right)}-\frac{17 \delta^{2} \hbar \omega^{3}}{24 c^{3} \pi^{2}\left(e^{\beta \hbar \omega}-1\right)}-\frac{\delta^{2} \beta \hbar^{2} \omega^{4} e^{\beta \hbar \omega}}{2 c^{3} \pi^{2}\left(e^{\beta \hbar \omega}-1\right)^{2}} \\
& -\frac{\delta^{2} \beta \hbar^{2} \omega^{4} e^{\beta \hbar \omega}}{c^{3} \pi^{2}\left(e^{\beta \hbar \omega}-1\right)^{3}}-\frac{\delta^{2} \beta \hbar^{2} \omega^{4} e^{\beta \hbar \omega}}{2 c^{3} \pi^{2}\left(e^{\beta \hbar \omega}-1\right)^{4}}
\end{aligned}
$$

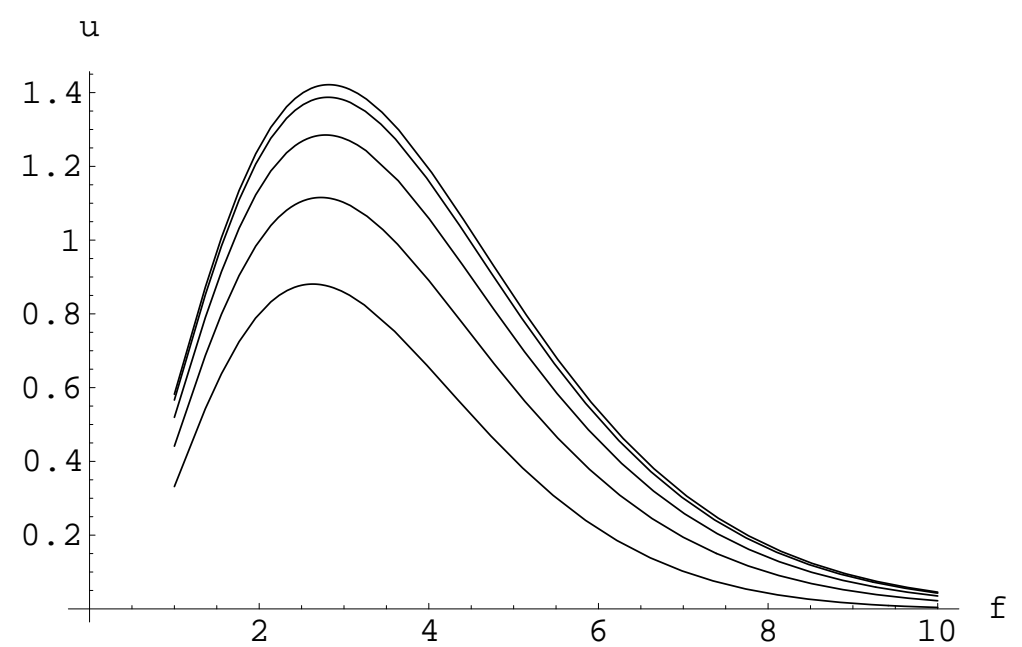

FIG.5 The Planck's formula of a deformed Bose gas ( $\operatorname{start}$ from up $q=1.0,1.1,1.2,1.3,1.4$, here we have used the notation $f=\beta \hbar \omega$.)

In Fig.5, we give a plot of the Planck's formula for the deformed Bose gas. The first term in equation(46) describes an ideal gas consisting of photons. The linearity of the equations of electrodynamics on commutative spacetime guarantees that photons do not interact with one another. However, the other terms in this equation denote 
interactions among photons or nonlinearity of equations of electrodynamics caused by noncommutative deformation of spacetime. At high frequencies $(\beta \hbar \omega \gg 1)$, we have

$$
\begin{aligned}
u(\omega, T)= & \frac{\hbar \omega^{3} e^{-\beta \hbar \omega}}{c^{3} \pi^{2}}-\frac{17 \delta^{2} \hbar \omega^{3} e^{-\beta \hbar \omega}}{24 c^{3} \pi^{2}}-\frac{\delta^{2} \beta \hbar^{2} \omega^{4} e^{-\beta \hbar \omega}}{2 c^{3} \pi^{2}} \\
& -\frac{\delta^{2} \beta \hbar^{2} \omega^{4} e^{-2 \beta \hbar \omega}}{c^{3} \pi^{2}}-\frac{\delta^{2} \beta \hbar^{2} \omega^{4} e^{-3 \beta \hbar \omega}}{2 c^{3} \pi^{2}} .
\end{aligned}
$$

This is the Wien's formula of the deformed Bose gas. We give a plot of the Wien's formula for different deformation parameters in Fig.6. In spite of a complex form of the Planck's formula and the Wien's formula, we note that the density of the spectral frequency distribution of the energy of the deformed Bose gas has a maximum at a frequency $\omega_{m}\left(\beta \hbar \omega_{m} \approx 2.8\right)$, which is roughly independent of deformation parameter. Thus the displacement law is still remained for the deformed Bose gas.

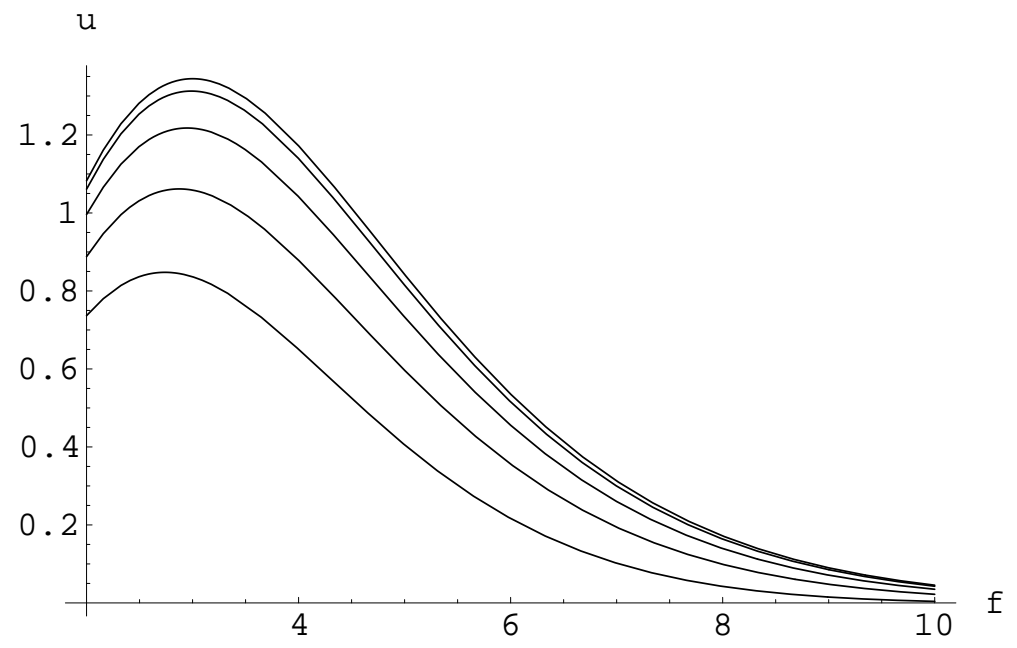

FIG.6 The Wien's formula of a deformed Bose gas ( start from up $q=1.0,1.1,1.2,1.3,1.4$, here we have used the notation $f=\beta \hbar \omega$ ).

In the opposite limiting case of low frequencies $(\beta \hbar \omega \ll 1)$, we get the Rayleigh-Jeans formula for the deformed Bose gas,

$$
\begin{aligned}
u(\omega, T)= & \frac{k T \omega^{2}}{c^{3} \pi^{2}}-\frac{17 \delta^{2} k T \omega^{2}}{24 c^{3} \pi^{2}}-\frac{\delta^{2} k T \omega^{2}}{2 c^{3} \pi^{2}}\left(\frac{\hbar \omega}{k T}+1\right) \\
& -\frac{\delta^{2}(k T)^{2} \omega}{c^{3} \pi^{2} \hbar}\left(\frac{\hbar \omega}{k T}+1\right)-\frac{\delta^{2}(k T)^{3}}{2 c^{3} \pi^{2} \hbar^{2}}\left(\frac{\hbar \omega}{k T}+1\right) .
\end{aligned}
$$


Fig.7 shows a graph corresponding to the Rayleigh-Jeans formula with different deformation parameters.

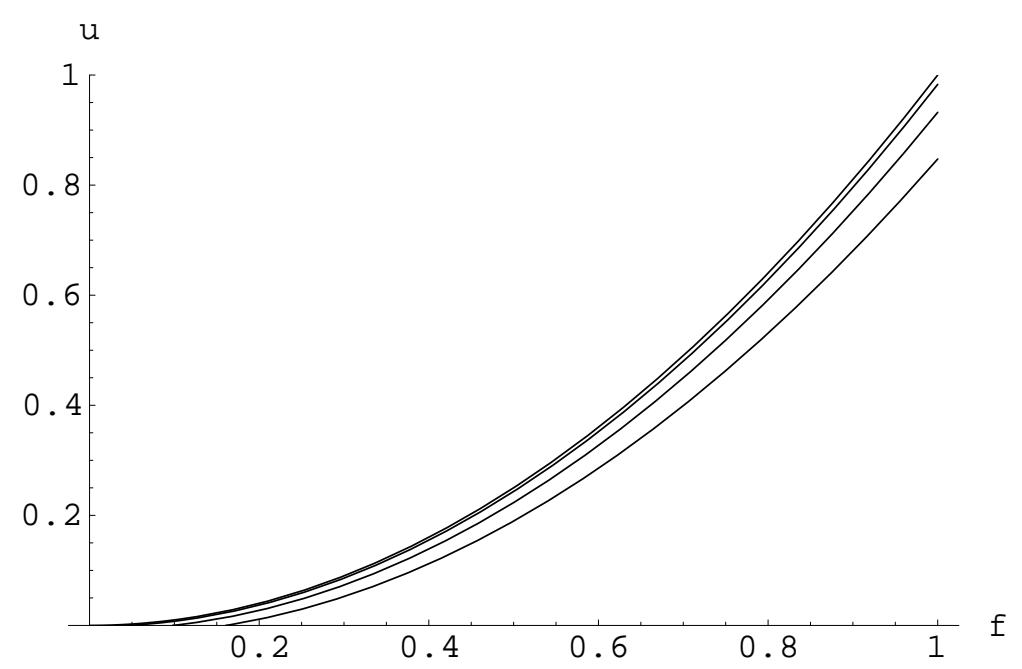

FIG.7 The Rayleigh-Jeans formula of a deformed Bose gas ( start from up $q=1.00,1.06,1.12,1.18$ ).

We obtain the Stefan-Boltzmann's law for the deformed Bose gas of the form,

$$
J=\frac{\pi^{2} k^{4}}{60 \hbar^{3} c^{2}} T^{4}-\frac{\delta^{2} k^{4}}{32 \pi^{2} \hbar^{3} c^{2}}\left(\frac{8 \pi^{2}}{3}+\frac{11 \pi^{4}}{15}+48 \zeta(3)\right) T^{4} .
$$

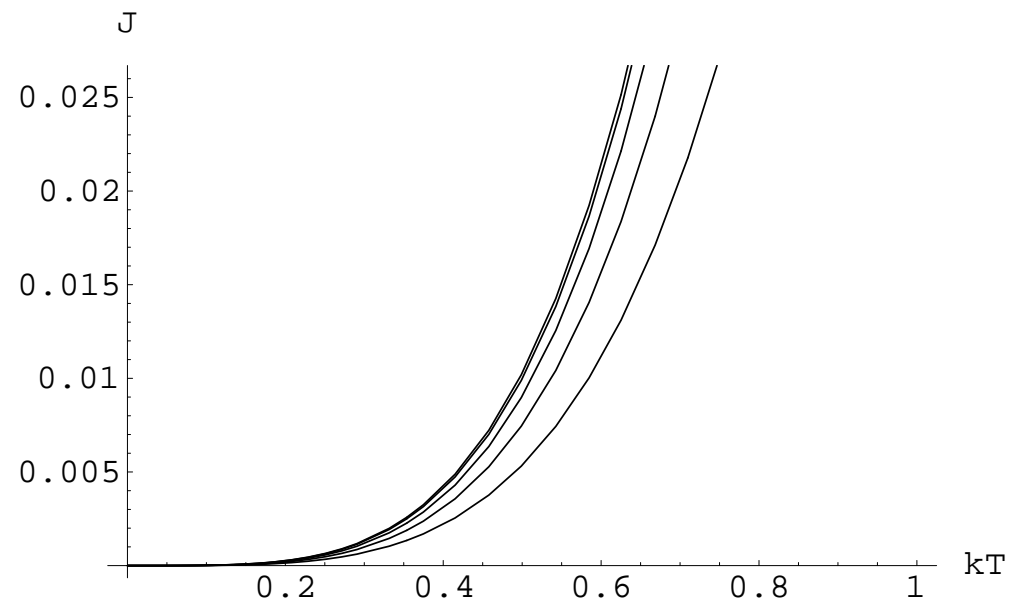

FIG.8 The Stefan-Boltzmann law of a deformed Bose gas ( start from up $q=1.0,1.1,1.2,1.3,1.4$ ).

Similar to the case of an ideal Bose gas, the total energy of the deformed Bose gas is proportional to the fourth power of the temperature. But the Stefan-Boltzmann's 
constant is effectively reduced by the noncommutative deformation of geometry. The fact gives also a signal that the deformed Bose gas is a strongly correlated system. We give a plot of the Stefan-Boltzmann's law for different deformation parameters in Fig.8.

In an adiabatic expansion (or compression) process, the entropy of the system is a constant and the valume and temperature are related by $V T^{3}=$ constant. From (42), we can get the equation of state,

$$
P V^{4 / 3}=c_{q}
$$

\section{Concluding Remarks}

The noncommutative geometry plays a very important role in the study of the Planck scale physics. Quantum field theory and superstring theory (or M theory) on noncommutative spacetime have been studied for a long time. It is well-known that the most realistic laboratory for testing the Planck scale physics is the early universe. The very early universe was opaque due to constant interchange of energy between matter and radiation. It is clear that the radiation field at the early universe can not be described well by an ideal Bose gas system. There should be strong correlations among photons at this stage of evolution of the universe. In fact, attention has been paid recently to the investigation on the deformed radiation dominated universe. Preliminary results show that spacetime quantization gives rise to inflation as the radiation temperature exceeds the Planck temperature. Of course, the study in this direction is only on its very beginning. Many efforts should be made to set up a self-contained inflation theory on noncommutative spacetime. We still have little knowledge on thermodynamics of the deformed radiation field.

From the linearity of the equations of electrodynamics on commutative spacetime, we know that photons do not interact with one another. The thermodynamics of the photonic field can be described well by an ideal Bose gas system. However, the

photons in the deformed radiation field have strong correlations among themselves. 
The strong correlations among the photons are caused by the nonlinearity of equations of electrodynamics on noncommutative spacetime. It is a difficult task to deal with the interactions among the photons. Numerous papers have been published[33]-[46] on the thermodynamics of the deformed photonic field. However, we still have not got a satisfactory distribution function of the deformed Bose gas.

In this paper, we have treated the deformed photonic field as a strongly correlated system by making use of the Green's function theory. Equations of motion of the Green's function for the deformed photonic field was set up. The Tyablikov procedure[57] was used to decouple these equations of motion. A general form of statistical distribution function for the deformed photonic field was got systematically. Based on this distribution function, we calculated the free energy, the entropy and the specific heat of the deformed photonic field in detail. The equation of state was obtained. We found that the Planck's formula for the deformed Bose gas is really different from the ordinary one. We got terms in Planck's formula, which correspond to the interactions among photons. Similar to the case of an ideal Bose gas, the total energy of the deformed Bose gas is proportional to the fourth power of the temperature. But the Stefan-Boltzmann's constant is effectively reduced by the noncommutative deformation of geometry. We noted that the density of the spectral frequency distribution of the energy of the deformed Bose gas has a maximum at a frequency $\omega_{m}\left(\beta \hbar \omega_{m} \approx 2.8\right)$, which is roughly independent of deformation parameter. Thus the displacement law is still

remained for the deformed Bose gas. The results obtained in the paper can be used to investigate properties of the early universe (e.g. inflation model on noncommutative spacetime). All of these works are in progress.

\section{Acknowledgements}

The work was supported in part by the Natural Science Foundation of China. 


\section{References}

[1] J. Wess, Int. J. Mod. Phys. A 12, 4997 (1997).

[2] N. Seiberg and E. Witten, JHEP 9909, 032 (1999).

[3] Z. Chang, Phys. Rev. D 61, 044009 (2000).

[4] T. Banks, W. Fischler, S. H. Shenker, and L. Susskind, Phys. Rev. D 55, 5112 (1997)

[5] A. Connes, M. R. Douglas, and A. Schwarz, JHEP 9802, 003 (1998).

[6] A. Connes, Noncommutative Geometry, Academic Press, London (1994).

[7] M. R. Douglas and N. A. Nekrasov, Rev. Mod. Phys. 73, 977 (2002).

[8] A. H. Guth, Phys. Rev. D23, 347 (1981).

[9] S. Hancock et al., Nature 367, 333 (1994).

[10] C. H. Lineweaver et al., Ap. J. 448, 482 (1995).

[11] A. Linde, D. Linde, and A. Mezhlumian, Phys. Rev. D49, 1783 (1994).

[12] A. Linde, "Lectures on inflationary cosmology", hep-th/9410082.

[13] J. Martin and R. H. Brandenberger, Phys. Rev. D63, 123501 (2001).

[14] J. C. Niemeyer, Phys. Rev. D63, 123502 (2001).

[15] J. Kowalski-Glikman, Phys. Lett. B499, 1 (2001).

[16] J. C. Niemeyer and R. Parentani, Phys. Rev. D64, 101301 (2001).

[17] R. H. Brandenberger and J. Martin, Mod. Phys. Lett A16, 999 (2001).

[18] J. A. Gu, P. M. Ho, and S. Ramgoolam, hep-th/0101058. 
[19] S. Alexander and J. Magueijo, hep-th/0104093.

[20] A. Kempf, Phys. Rev. D63, 083514 (2001).

[21] C. S. Chu, B. R. Greene, and G. Shiu, Mod. Phys. Lett. A16, 2231 (2001).

[22] A. Kempf and J. C. Niemeyer, Phys. Rev. D64, 103501 (2001).

[23] R. Easther, B. R. Greene, W. H. Kinney, and G. Shiu, Phys. Rev. D64, 103502 (2001).

[24] S. Alexander, R. Brandenberger, and J. Magueijo, hep-th/0108190.

[25] M. Chaichian, D. Ellinas, and P. Kulish, Phys. Rev. Lett. 65, 980 (1990).

[26] Z. Chang, Phys. Rev. A46, 5865 (1992).

[27] Z. Chang, Phys. Rev. A47, 5017 (1993).

[28] E. Celeghini, M. Rasetti, and G. Vitiello, Phys. Rev. Lett. 66, 2056 (1991).

[29] Z. Chang, Phys. Rev. A46, 5860 (1992).

[30] Z. Chang, H. Y. Guo, and H. Yan, Phys. Lett. A156, 192 (1991).

[31] Z. Chang and H. Yan, Phys. Rev. A43, 6043 (1991).

[32] Z. Chang, Phys. Rept. 262, 137 (1995).

[33] P. Angelopoulou et al., J. Phys. A: Math. Gen. 27, L605 (1994).

[34] M. A. Martín-Delgado, J. Phys. A: Math. Gen. 24, L1285 (1991).

[35] S. Vokos and C. Zachos, Mod. Phys. Lett. A9, 1 (1994).

[36] J. A. Tuszyński, J. L. Rubin, J. Meyer, and M. Kibler, Phys. Lett. A175, 173 (1993). 
[37] M. L. Ge and G. Su, J. Phys. A: Math. Gen. 24, L721 (1991).

[38] J. L. Birman, Phys. Lett. A167, 363 (1992).

[39] Y. J. Ng, J. Phys. A: Math. Gen. 23, 1023 (1990).

[40] J. W. Goodison and D. J. Toms, Phys. Lett. A195, 38 (1994); 198, 471 (1995).

[41] R. A. Campos, Phys. Lett. A184, 173 (1994).

[42] S. L. Dalton and A. Inomata, Phys. Lett. A199, 315 (1995).

[43] G. Su and M. L. Ge, Phys. Lett. A173, 17 (1993).

[44] D. I. Fivel, Phys. Rev. Lett. 65, 3361 (1990).

[45] A. Inomata, Phys. Rev. A52, 932 (1995).

[46] M. R. Ubriaco, Phys. Rev. E55, 291 (1997).

[47] A. J. Macfarlane, J. Phys. A: Math. Gen. 22, 4581 (1989).

[48] L. C. Biedenharn, J. Phys. A: Math. Gen. 22, L873 (1989).

[49] C. P. Sun and H. C. Fu, J. Phys. A: Math. Gen. 22, L983 (1989).

[50] X. C. Song, J. Phys. A: Math. Gen. 23, L821 (1990).

[51] Z. Chang, W. Chen, and H. Y. Guo, J. Phys. A: Math. Gen. 23, 4185 (1990).

[52] Z. Chang, W. Chen, H. Y. Guo, and H. Yan, J. Phys. A: Math. Gen. 23, 5371 (1990).

[53] Z. Chang, W. Chen, H. Y. Guo, and H. Yan, J. Phys. A: Math. Gen. 24, 1427 (1991).

[54] Z. Chang, S. M. Fei, H. Y. Guo, and H. Yan, J. Phys. A: Math. Gen. 24, 5435 (1991). 
[55] D. N. Zubarev, Sov. Phys. -Usp. 3, 320 (1960).

[56] Z. Chang, Ann. Phys. (N.Y.) 271, 233 (1999).

[57] S. V. Tyablikov, Ukrain. Mat. Zh. 11, 287 (1959). 
BIHEP-TH-2002-21

\title{
Thermodynamics of a deformed Bose gas
}

\author{
Zhe Chang ${ }^{1}$ and Shao-Xia Chen ${ }^{2}$ \\ Institute of High Energy Physics, Chinese Academy of Sciences \\ P.O.Box 918(4), 100039 Beijing, China
}

\begin{abstract}
By making use of the double-time Green function technique, we study thermodynamics of a deformed Bose gas, which describes well properties of density intensive photonic gas and radiation fields of the early universe. General form of statistical distribution function is obtained. We show explicitly the expression of the distribution function in some limitation cases. The free energy, equation of state, specific heat and other thermodynamic properties of the deformed Bose gas are presented.
\end{abstract}

PACS numbers: 05.30.-d, 05.10.-a, 98.80.Cq, 05.70.-a, 51.30.+i

\section{Introduction}

There are two main motivations to study a deformed many-body system. The first one comes from fundamental physics. It is well-known that the noncommutative geometry plays a very important role in unravelling the properties of the Planck scale physics. It has for a long time been suspected that the noncommutative spacetime might be a realistic picture of how spacetime behaves near the Planck scale[1]. In fact, the

\footnotetext{
${ }^{1}$ changz@mail.ihep.ac.cn

${ }^{2}$ ruxanna@mail.ihep.ac.cn
} 
noncommutative geometry naturally enters the theory of open string in a background $B$-field[2]. In particular, the noncommutative geometry makes the holography[3] (e.g. the AdS/CFT correspondence) of a higher dimensional quantum system of gravity and lower dimensional theory possible. A kind of special regularization with exponentially increasing spacetime cutoff has been introduced based on the noncommutative geometry. A very small displacement of the noncommutative deformation parameter from its classical value reduces sharply the entropy of quantum system of gravity. An adequately adopted noncommutative deformation of geometry makes the holography of a higher dimensional quantum system of gravity and lower dimensional theory possible. It was also discovered that simple limits of $M$ theory and superstring theory lead directly to the noncommutative gauge field theory $[4,5]$. The noncommutative field theory has been intensively studied in the past two decads $[6,7]$. The most realistic laboratory for testing the Planck scale physics is the early universe and the black hole physics. From the standard picture of cosmology, the universe started from a very hot, dense phase about 15 billion years ago in the big bang. The very early universe was opaque due to the constant interchange of energy between matter and radiation. Several problems of standard big bang cosmology, such as the horizon, homogeneity, entropy and flatness problems, can be explained under the assumption that the very early universe underwent a period of extremely rapid inflation[8], driven by the potential of some assumed inflation fields. In particular, the inflation scenario is also able to explain the observed perturbations in the cosmic microwave background radiation $[9,10]$, namely as originating ultimately from quantum fluctuations of the inflation field. However, in most of the current models of inflation, the physical length of perturbations was much smaller than the Planck length at the beginning of inflation[11, 12]. It is then of interest to investigate whether the predictions of the spectrum of cosmological perturbations are sensitive to the unknown super-Planck-scale physics[13]-[16]. Recent studies[17]-[24] by physicists and astrophysicists show that without strange inflation fields but just the noncommutative geometry and the deformed radiation dominated universe subject 
to the noncommutative spacetime quantization indeed give rise to inflation as the radiation temperature exceeds the Planck temperature. Based on the noncommutative geometry and the deformed radiation, one can even get an alternative explanation for the horizon, homogeneity, entropy and flatness problems in the standard cosmology.

Another motivation comes from quantum optics as well as molecular and atomic spectroscopy. A fascinating development of quantum optics in last two decades has taken place within two specific areas, one concerning the study of nonclassical states of light, and the other studying the interaction of light with matter. The nonclassical states of light offer the possibility of the so-called reduction of quantum noise below the standard limit, determined by the Heisenberg's uncertainty relation. Study on interaction of light with matter shows that the old Bohr quantum jumps are real. Dynamics of deformed photonic field interacting with matter presented many interesting properties both in the two important areas[25]-[29]. Great success has also been made by making use of a deformed oscillator to study molecular spectrum[30]-[32].

In this paper, we study thermodynamics of a deformed Bose gas, and in particular, of a deformed radiation field. On the topic, many papers have been published in more than ten years[33]-[46]. However, we still have not a general form of statistical distribution function of the deformed Bose gas, which should reduce to the Bose-Einstein distribution function for an ideal Bose gas in the $q=1$ limit. In this situation, it is difficult to investigate systematically the influence of the deformation on thermodynamic characteristics of a Bose system, which is interesting in fundamental physics and other subjects as discussed above. For this purpose, by making use of the double-time Green function technique, we obtain an algebra equation, which should be satisfied by the distribution function of a deformed Bose gas. The equation for distribution function can not be solved exactly in general. The situation is similar to the case of magnetic lattices. From this point of view, a deformed Bose gas is really a strongly correlated system. In the case of $q \rightarrow 1$, we give a useful expression of the distribution function for a deformed Bose gas. Thermodynamic properties, such as internal energy, specific 
heat, free energy and equation of state, are discussed in detail.

The paper is organized as follows. In section 2, a deformed Bose gas is discussed. We get the distribution function for a deformed Bose gas by using the double-time Green function technique in section 3. In section 4, we calculate some thermodynamic quantities for a deformed photon gas and compare them with those for the ideal Bose gas. Concluding remarks are given in section 5 .

\section{A deformed Bose gas system}

The Lagrange density of a scalar field $\phi(x)$ with mass $m$ reads

$$
\mathcal{L}(x)=\frac{\hbar^{2}}{2} \frac{\partial \phi}{\partial x_{\mu}} \frac{\partial \phi}{\partial x^{\mu}}-\frac{1}{2} m^{2} c^{2} \phi^{2} .
$$

The Euler-Lagrange equation immediately leads to the Klein-Gordon equation

$$
\left(\hbar^{2} \frac{\partial}{\partial x_{\mu}} \frac{\partial}{\partial x^{\mu}}+m^{2} c^{2}\right) \phi(x)=0 .
$$

The canonically conjugate field is

$$
\pi(x)=\frac{\partial \mathcal{L}}{\partial \dot{\phi}(x)},
$$

which leads to the Hamilton density

$$
\mathcal{H}(x)=\frac{1}{2}\left(\frac{1}{\hbar^{2}} \pi^{2}(x)+(\hbar \nabla \phi(x))^{2}+m^{2} c^{2} \phi^{2}(x)\right) .
$$

The Fourier decomposition of the scalar field is of the form,

$$
\begin{aligned}
& \phi(x)=\int \frac{d^{3} k c}{\sqrt{(2 \pi)^{3} 2 \hbar \omega_{k}}}\left(a(k) e^{-i k x}+a^{\dagger}(k) e^{i k x}\right), \\
& \pi(x)=\int \frac{d^{3} k i \hbar^{2} \omega_{k}}{\sqrt{(2 \pi)^{3} 2 \hbar \omega_{k}}}\left(-a(k) e^{-i k x}+a^{\dagger}(k) e^{i k x}\right),
\end{aligned}
$$

where we have used the notation $\omega_{k}=\frac{c}{\hbar} \sqrt{(\hbar \mathbf{k})^{2}+m^{2} c^{2}}$. The canonical quantization of the scalar field reads

$$
[\hat{\phi}(\mathbf{x}, t), \hat{\pi}(\mathbf{y}, t)]=i \hbar c \delta^{3}(\mathbf{x}-\mathbf{y})
$$


In terms of the Fourier modes $\hat{a}(k)$ and $\hat{a}^{\dagger}(k)$, the commutation relations can be rewritten into the form

$$
\left[\hat{a}(k), \hat{a}^{\dagger}\left(k^{\prime}\right)\right]=\delta^{3}\left(\mathbf{k}-\mathbf{k}^{\prime}\right)
$$

It is not difficult to express the Hamiltonian in terms of these Fourier modes,

$$
\hat{H}=\frac{1}{2} \int d^{3} k \hbar \omega_{k}\left(\hat{a}(k) \hat{a}^{\dagger}(k)+\hat{a}^{\dagger}(k) \hat{a}(k)\right)
$$

In the quantum field theory, the vacuum state is defined as follows

$$
\hat{a}(k)|0\rangle=0
$$

And the $N$-particle Fock space can be written down directly as

$$
\left|n\left(k_{1}\right), n\left(k_{2}\right), \cdots, n\left(k_{m}\right)\right\rangle=\frac{\left(\hat{a}^{\dagger}\left(k_{1}\right)\right)^{n\left(k_{1}\right)}}{\sqrt{n\left(k_{1}\right) !}} \frac{\left(\hat{a}^{\dagger}\left(k_{2}\right)\right)^{n\left(k_{2}\right)}}{\sqrt{n\left(k_{2}\right) !}} \cdots \frac{\left(\hat{a}^{\dagger}\left(k_{m}\right)\right)^{n\left(k_{m}\right)}}{\sqrt{n\left(k_{m}\right) !}}|0\rangle
$$

To measure how many particles of a certain momentum exist in the field system, one can introduce the particle number operator $\hat{N}(k)$,

$$
\hat{N}(k)=\hat{a}^{\dagger}(k) \hat{a}(k)
$$

It is easy to check that

$$
\hat{N}\left(k_{i}\right)\left|n\left(k_{1}\right), n\left(k_{2}\right), \cdots, n\left(k_{m}\right)\right\rangle=n\left(k_{i}\right)\left|n\left(k_{1}\right), n\left(k_{2}\right), \cdots, n\left(k_{m}\right)\right\rangle
$$

Thus, a field can be seen as a many-body system that consists of free harmonic oscillators with different momentum. Properties of the field system can be described exactly by the Weyl-Heisenberg algebra,

$$
\begin{aligned}
& {\left[\hat{a}, \hat{a}^{\dagger}\right]=1,} \\
& {[\hat{N}, \hat{a}]=-\hat{a}, \quad\left[\hat{N}, \hat{a}^{\dagger}\right]=\hat{a}^{\dagger},}
\end{aligned}
$$

with the Fock space $|n\rangle=\frac{\left(\hat{a}^{\dagger}\right)^{n}}{\sqrt{n !}}|0\rangle$ as its representation. All properties, in particular, the thermodynamic properties of the many-body system can be investigated systematically, by making use of the Hamiltonian (8) and the Weyl-Heisenberg algebra (13). 
The natural quantum of the quantum field theory on noncommutative geometry is the so-called deformed harmonic oscillator. On the topic of deformed oscillators, a lot of papers have been published[47]-[49]. Similar to the case of an ordinary quantum field system, the many-body properties of a noncommutative quantum field system can be described by a collection of the deformed harmonic oscillators,

$$
\hat{H}_{q}=\frac{1}{2} \sum_{\mathbf{k}} \hbar \omega_{k}\left(\hat{a}_{q}(k) \hat{a}_{q}^{\dagger}(k)+\hat{a}_{q}^{\dagger}(k) \hat{a}_{q}(k)\right) .
$$

The deformed harmonic oscillator is related with the simple harmonic oscillator as follows[50],

$$
\hat{a}_{q}=\sqrt{\frac{[\hat{N}]_{q}}{\hat{N}}} \hat{a}, \quad \hat{a}_{q}^{\dagger}=\hat{a}^{\dagger} \sqrt{\frac{[\hat{N}]_{q}}{\hat{N}}},
$$

where we have used the notation $[x]_{q}=\frac{q^{x}-q^{-x}}{q-q^{-1}}$.

The deformed Weyl-Heisenberg algebra reads

$$
\begin{aligned}
& \hat{a}_{q} \hat{a}_{q}^{\dagger}-q \hat{a}_{q}^{\dagger} \hat{a}_{q}=q^{-\hat{N}}, \\
& {\left[\hat{N}, \hat{a}_{q}^{\dagger}\right]=\hat{a}_{q}^{\dagger}, \quad\left[\hat{N}, \hat{a}_{q}\right]=-\hat{a}_{q} .}
\end{aligned}
$$

The representation of the deformed Weyl-Heisenberg algebra is obtained by constructing the Fock space,

$$
\begin{aligned}
& |n\rangle_{q}=\frac{\left(\hat{a}_{q}^{\dagger}\right)^{n}}{\sqrt{[n]_{q} !}}|0\rangle, \\
& \hat{a}_{q}|n\rangle_{q}=\sqrt{[n]_{q}}|n-1\rangle_{q}, \quad \hat{a}_{q}^{\dagger}|n\rangle_{q}=\sqrt{[n+1]_{q}}|n+1\rangle_{q},
\end{aligned}
$$

where $[n]_{q} ! \equiv[n]_{q}[n-1]_{q} \cdots[2]_{q}[1]_{q}$. It is easy to check that one still can measure how many particles of a certain momentum exist in the deformed field system by making use of the particle number operator $\hat{N}(k)$,

$$
\hat{N}\left(k_{i}\right)\left|n\left(k_{1}\right), n\left(k_{2}\right), \cdots, n\left(k_{m}\right)\right\rangle_{q}=n\left(k_{i}\right)\left|n\left(k_{1}\right), n\left(k_{2}\right), \cdots, n\left(k_{m}\right)\right\rangle_{q}
$$

The Hamiltonian of a deformed harmonic oscillator[51]-[54] can be written as

$$
\hat{H}_{q}=\frac{\hbar \omega}{2}\left([\hat{N}]_{q}+[\hat{N}+1]_{q}\right)
$$

Below we will calculate the distribution function of a deformed harmonic oscillator system by making use of the double-time Green function technique. 


\section{Distribution function}

The double-time Green function (retarded or advanced) for a Bose system[55, 56] is generally defined as

$$
G_{\rho}\left(t-t^{\prime}\right)=-\frac{i}{2}\left((\rho+1) \theta\left(t-t^{\prime}\right)+(\rho-1) \theta\left(t^{\prime}-t\right)\right)\left\langle\left[\hat{A}(t), \hat{B}\left(t^{\prime}\right)\right]\right\rangle
$$

where we have used the notations,

$$
\begin{aligned}
& \hat{A}(t) \equiv \exp \left(\frac{i H t}{\hbar}\right) \hat{A} \exp \left(\frac{-i H t}{\hbar}\right), \\
& \langle\hat{O}\rangle=Z^{-1} \operatorname{Tr}\left(e^{-\beta H} \hat{O}\right), \quad Z=\operatorname{Tr}\left(e^{-\beta H}\right),
\end{aligned}
$$

here $\rho=1$ for the retarded one and $\rho=-1$ for the advanced one.

In Eq.(20), setting

$$
\hat{A}(t) \equiv \hat{a}(t), \quad \hat{B}\left(t^{\prime}\right) \equiv \hat{a}^{\dagger}\left(t^{\prime}\right), \quad \rho=1,
$$

(the retarded Green function is assumed), we get

$$
G_{R}\left(t-t^{\prime}\right)=-i \theta\left(t-t^{\prime}\right)\left\langle\hat{a}(t) \hat{a}^{\dagger}\left(t^{\prime}\right)-\hat{a}^{\dagger}\left(t^{\prime}\right) \hat{a}(t)\right\rangle
$$

The equation of motion of the retarded Green function reads

$i \hbar \frac{\partial}{\partial t} G_{R}\left(t-t^{\prime}\right)=\hbar\left(\delta\left(t-t^{\prime}\right)\left\langle\hat{a}(t) \hat{a}^{\dagger}\left(t^{\prime}\right)-\hat{a}^{\dagger}\left(t^{\prime}\right) \hat{a}(t)\right\rangle+\theta\left(t-t^{\prime}\right)\left\langle\frac{d \hat{a}(t)}{d t} \hat{a}^{\dagger}\left(t^{\prime}\right)-\hat{a}^{\dagger}\left(t^{\prime}\right) \frac{d \hat{a}(t)}{d t}\right\rangle\right)$.

The Heisenberg equation for the operator $\hat{a}(t)$

$$
i \hbar \frac{d \hat{a}(t)}{d t}=[\hat{a}(t), \hat{H}(t)]
$$

gives,

$$
\frac{d \hat{a}(t)}{d t}=-i \frac{\omega}{2}\left(q^{\hat{N}+1}+q^{-(\hat{N}+1)}\right) \hat{a}(t)
$$

Then, we have

$$
i \hbar \frac{\partial}{\partial t} G_{R}\left(t-t^{\prime}\right)=\hbar \delta\left(t-t^{\prime}\right)+\frac{\hbar \omega}{2}\left(q^{\langle\hat{N}+1\rangle}+q^{-\langle\hat{N}+1\rangle}\right) G_{R}\left(t-t^{\prime}\right)
$$


Making use of the Fourier transformation

$$
G_{R}\left(t-t^{\prime}\right)=\int_{-\infty}^{\infty} \frac{d E}{2 \pi \hbar} G_{R}(E) e^{-i E\left(t-t^{\prime}\right) / \hbar}
$$

we get

$$
G_{R}(E)=\frac{\hbar}{E-\frac{\hbar \omega\left(q^{\langle\hat{N}+1\rangle}+q^{-\langle\hat{N}+1\rangle}\right)}{2}} .
$$

The spectrum theorem of Green functions tells us

$$
\left\langle\hat{a}^{\dagger}\left(t^{\prime}\right) \hat{a}(t)\right\rangle=i \int_{-\infty}^{\infty} d E \frac{\left(G_{R}\left(E+i 0^{\dagger}\right)-G_{R}\left(E-i 0^{\dagger}\right)\right) e^{-i E\left(t-t^{\prime}\right) / \hbar}}{2 \pi \hbar\left(e^{\beta E}-1\right)} .
$$

Then, we can write formally the distribution function of a deformed Bose gas as

$$
n(\varepsilon)=\langle\hat{N}\rangle=\frac{1}{\exp \left(\frac{\hbar \omega \beta\left(q^{\langle\hat{N}+1\rangle}+q^{-\langle\hat{N}+1\rangle}\right)}{2}\right)-1} .
$$

Evidently, in the case of $q \rightarrow 1, n(\varepsilon)$ recovers the familiar Bose-Einstein distribution function. In principle, all interesting properties of a deformed Bose gas can be obtained by solving this equation for distribution function. This is to be one of the most advantageous features of the Green function approach.

Now we try to solve Eq.(28) to gain an insight into the influence of the deformation on the distribution function and to compare the deformed distribution function with the Bose-Einstein distribution function.

Expanding the right hand side of Eq.(28) in the neighborhood of $q=1$ to the second order, we get an exactly solvable equation for the deformed distribution function. The solution is of the form,

$$
\begin{aligned}
n(\varepsilon)= & -\frac{1}{\delta^{2} \beta \varepsilon e^{\beta \varepsilon}}\left(\delta^{2} \beta \varepsilon e^{\beta \varepsilon}+\left(e^{\beta \varepsilon}-1\right)^{2}\right) \\
& +\frac{1}{\delta^{2} \beta \varepsilon e^{\beta \varepsilon}} \sqrt{\left(\delta^{2} \beta \varepsilon e^{\beta \varepsilon}+\left(e^{\beta \varepsilon}-1\right)^{2}\right)^{2}-\delta^{2} \beta \varepsilon e^{\beta \varepsilon}\left(\delta^{2} \beta \varepsilon e^{\beta \varepsilon}-2\left(e^{\beta \varepsilon}-1\right)\right)},
\end{aligned}
$$

where we have used the notation $\delta \equiv q-1$ for short.

We note that it is difficult to compare the distribution function of the deformed Bose gas, Eq.(29), with the Bose-Einstein distribution function directly. For the aim, 
we rewrite Eq.(29) as follows,

$$
n(\varepsilon)=\frac{\left(e^{\beta \varepsilon}-1\right)-2^{-1} \delta^{2} \beta \varepsilon e^{\beta \varepsilon}}{\left(e^{\beta \varepsilon}-1\right)^{2}+\delta^{2} \beta \varepsilon e^{\beta \varepsilon}}-\frac{\delta^{2} \beta \varepsilon e^{\beta \varepsilon}\left(\delta^{2} \beta \varepsilon e^{\beta \varepsilon}-2\left(e^{\beta \varepsilon}-1\right)\right)^{2}}{\left(2 \delta^{2} \beta \varepsilon e^{\beta \varepsilon}+2\left(e^{\beta \varepsilon}-1\right)^{2}\right)^{3}} .
$$

One can further expand the distribution function into a series over deformation parameter $\delta$,

$$
n(\varepsilon)=\frac{1}{e^{\beta \varepsilon}-1}-\delta^{2}\left(\frac{\beta \varepsilon e^{\beta \varepsilon}}{2\left(e^{\beta \varepsilon}-1\right)^{2}}+\frac{\beta \varepsilon e^{\beta \varepsilon}}{\left(e^{\beta \varepsilon}-1\right)^{3}}+\frac{\beta \varepsilon e^{\beta \varepsilon}}{2\left(e^{\beta \varepsilon}-1\right)^{4}}\right) .
$$

From the above form of the distribution function of the deformed Bose gas, we see clearly that, at the case of $q=1$, the Bose-Einstein distribution is recovered. To further investigate the effect of deformation on the distribution function, we present a plot of the distribution function in Fig.1.

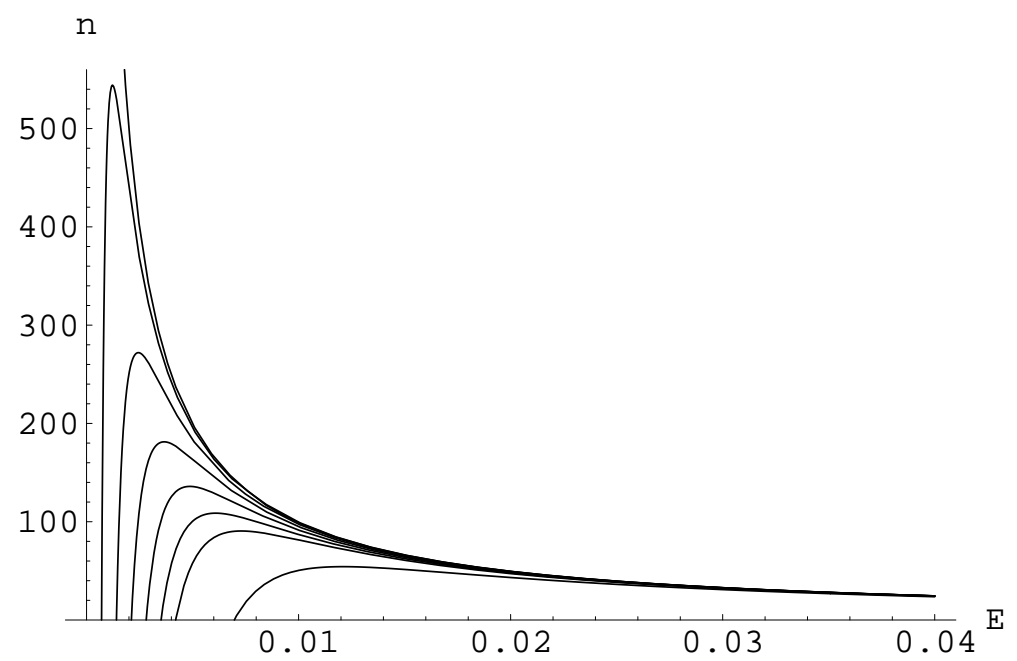

FIG.1 The distribution function of a deformed Bose gas

( start from up $q=1.000,1.001,1.002,1.003,1.004,1.005,1.006,1.010$, here we have used the notation $\beta \varepsilon=E$.)

To describe behaviors of the distribution function of the deformed Bose gas near the ground state more accurately, we give another plot of the deformed distribution function in Fig.2. 


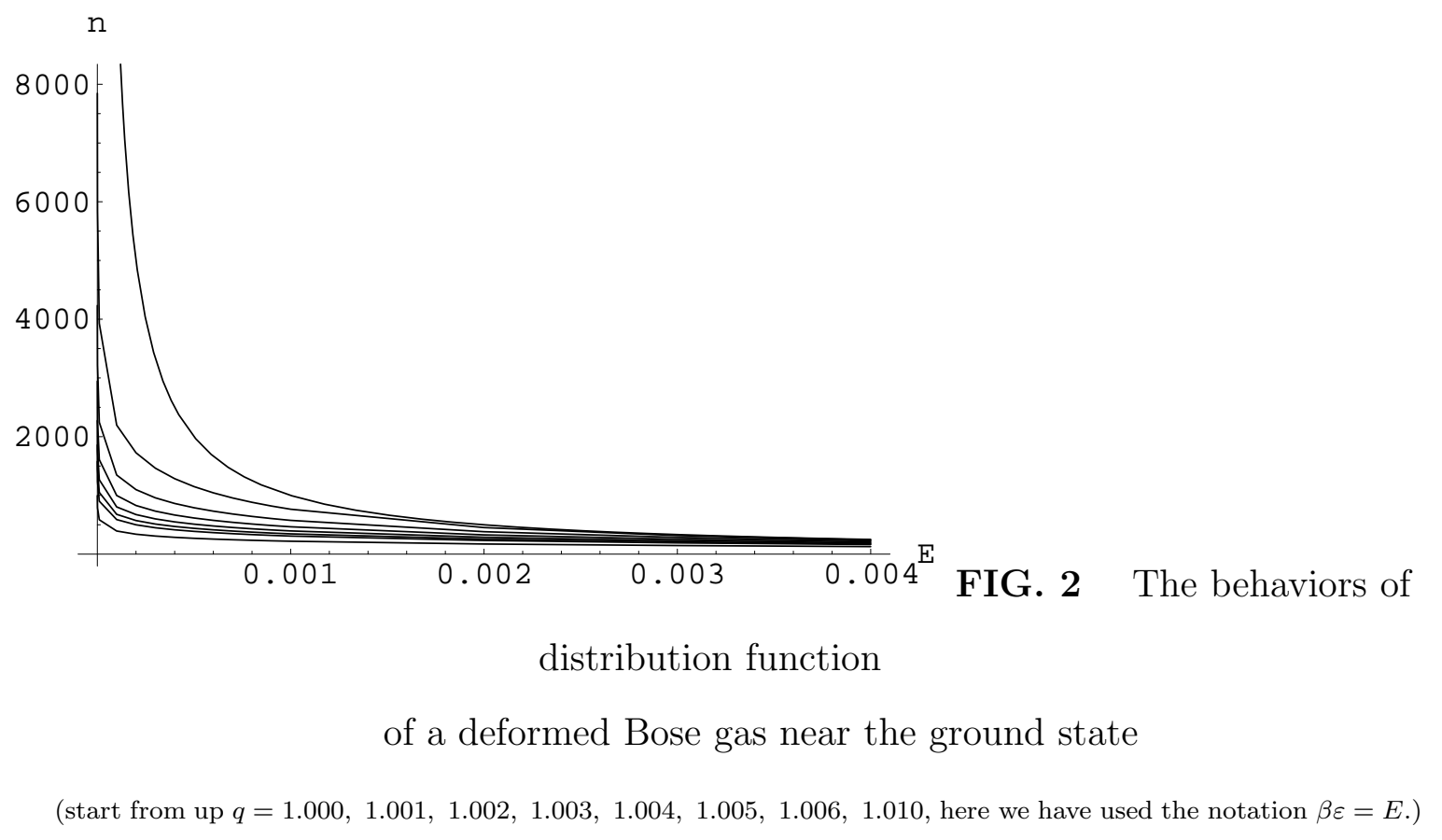

\section{Thermodynamic properties}

In this section, we concentrate on the study of thermodynamic properties of a deformed photonic field. As being well-known, the dispersion relation of a photon is

$$
\epsilon=c p
$$

We know that a photonic field can be described by a collection of simple harmonic oscillators and have relation

$$
\hat{H}=\hbar \omega\left(\hat{N}+\frac{1}{2}\right)
$$

One can then read out that

$$
p=\frac{\hbar \omega}{c}\left(n+\frac{1}{2}\right) .
$$

This relation and the Hamiltonian (19) give the dispersion relation of a deformed Bose gas,

$$
\varepsilon=\frac{1}{2} \hbar \omega\left(\left[\frac{c}{\hbar \omega} p+\frac{1}{2}\right]_{q}+\left[\frac{c}{\hbar \omega} p-\frac{1}{2}\right]_{q}\right) .
$$


Expanding the right hand side of (35) in the neighborhood of $q=1$ to the second order, we get

$$
\varepsilon=c p-\frac{\delta^{2}}{24} c p+\frac{\delta^{2}}{6 \hbar^{2} \omega^{2}} c^{3} p^{3} .
$$

The density of energy levels is as follows,

$$
\begin{aligned}
a(\varepsilon)= & \frac{8 \pi V}{3 h^{3} c^{3}} \cdot \frac{3 \sqrt{3 B} \varepsilon+\sqrt{4 A^{3}+27 B \varepsilon^{2}}}{4 B \sqrt{4 A^{3}+27 B \varepsilon^{2}}} \cdot \frac{\left(-2 \times 6^{1 / 3} A+\left(18 \sqrt{B} \varepsilon+2 \sqrt{3\left(4 A^{3}+27 B \varepsilon^{2}\right)}\right)^{2 / 3}\right)^{2}}{\left(18 \sqrt{B} \varepsilon+2 \sqrt{3\left(4 A^{3}+27 B \varepsilon^{2}\right)}\right)^{1 / 3}} \\
& \times \frac{9 \sqrt{B} \varepsilon+\sqrt{3\left(4 A^{3}+27 B \varepsilon^{2}\right)}+6^{1 / 3} A\left(18 \sqrt{B} \varepsilon+2 \sqrt{3\left(4 A^{3}+27 B \varepsilon^{2}\right)}\right)^{1 / 3}}{\left(9 \sqrt{B} \varepsilon+\sqrt{3\left(4 A^{3}+27 B \varepsilon^{2}\right)}\right)^{2}},
\end{aligned}
$$

where we have used the notations $A \equiv\left(1-\frac{\delta^{2}}{24}\right) c$ and $B \equiv \frac{\delta^{2}}{6 \hbar^{2} \omega^{2}} c^{3}$.

The mean value of the total energy of the system has the form,

$$
\bar{E}=\int_{0}^{\infty} \varepsilon n(\varepsilon) a(\varepsilon) d \varepsilon
$$

To calculate $\bar{E}$, we expand the right hand side of (37) to the third order in the neighborhood of $q=1$ and rewrite the density of energy levels as the form

$$
a(\varepsilon)=\frac{8 \pi V}{3 h^{3} c^{3}}\left(3-\frac{17}{8} \delta^{2}\right) \varepsilon^{2} .
$$

Then we get

$$
\bar{E}=\frac{8 \pi^{5} V}{15} \frac{(k T)^{4}}{(h c)^{3}}-\pi V \delta^{2}\left(\frac{8 \pi^{2}}{3}+\frac{11 \pi^{4}}{15}+48 \zeta(3)\right) \frac{(k T)^{4}}{(h c)^{3}},
$$

where $\zeta(n)$ is the Riemann zeta function.

We can further calculate the grand partition function $\ln \Xi$ through the thermodynamic relation $\bar{E}=-\frac{\partial}{\partial \beta} \ln \Xi$,

$$
\ln \Xi=\frac{8 \pi^{5} V}{45 h^{3} c^{3}}(k T)^{3}-\frac{\pi V \delta^{2}}{h^{3} c^{3}}\left(\frac{8 \pi^{2}}{9}+\frac{11 \pi^{4}}{45}+16 \zeta(3)\right)(k T)^{3} .
$$

It is a straightforward calculation to obtain thermodynamic functions by making use of the grand partition function. The pressure of the deformed Bose gas is

$$
P=\frac{1}{\beta} \frac{\partial}{\partial V} \ln \Xi=\frac{8 \pi^{5}}{45 h^{3} c^{3}}(k T)^{4}-\frac{\pi \delta^{2}}{h^{3} c^{3}}\left(\frac{8 \pi^{2}}{9}+\frac{11 \pi^{4}}{45}+16 \zeta(3)\right)(k T)^{4} .
$$


The free energy of the deformed Bose gas is of the form,

$$
F=-\frac{8 \pi^{5} V}{45} \frac{(k T)^{4}}{(h c)^{3}}+\pi V \delta^{2}\left(\frac{8 \pi^{2}}{9}+\frac{11 \pi^{4}}{45}+16 \zeta(3)\right) \frac{(k T)^{4}}{(h c)^{3}} .
$$

The entropy of the deformed Bose gas is as

$$
S=\frac{U-F}{T}=\frac{32 \pi^{5}}{45} \frac{k^{4} T^{3}}{h^{3} c^{3}} V-\pi \delta^{2}\left(\frac{32 \pi^{2}}{9}+\frac{44 \pi^{4}}{45}+64 \zeta(3)\right) \frac{k^{4} T^{3}}{h^{3} c^{3}} V .
$$

The first term in the above equation is just the entropy of an ordinary Bose gas. And the terms following it denote the effect of noncommutative deformation of geometry on the entropy. It is clear that the effect can not be ignored at least in the high temperature region, where the early universe lived. We give a plot of the entropy vs temperature for different deformation parameters in Fig. 3.

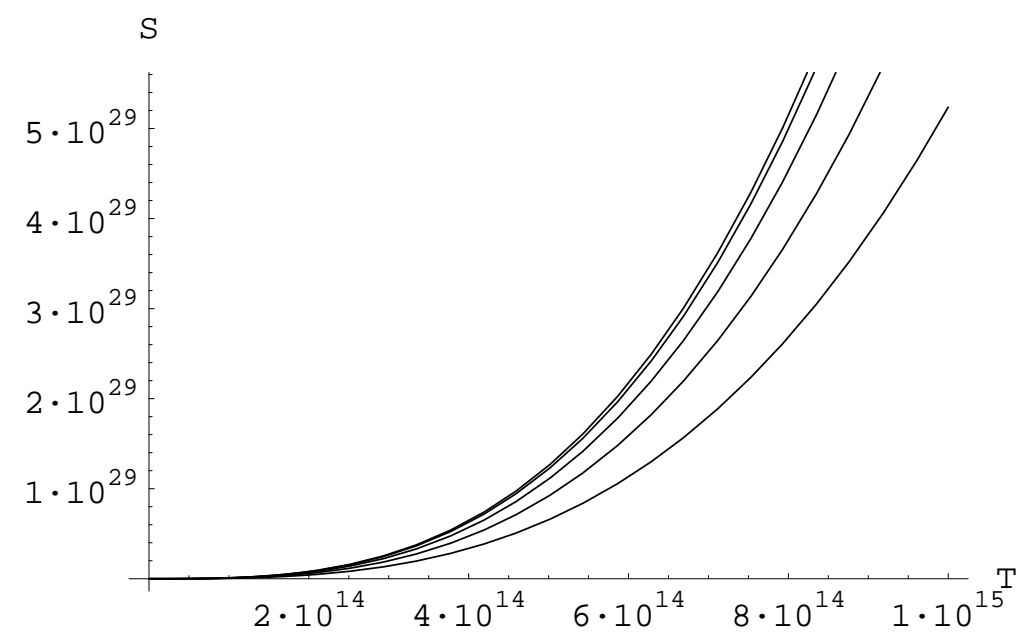

FIG.3 The entropy per unit volume of a deformed Bose gas ( start from up $q=1.0,1.1,1.2,1.3,1.4$ ).

Finally, the specific heat of the deformed Bose gas is

$$
C_{V}=T\left(\frac{\partial S}{\partial T}\right)_{V}=\frac{32 \pi^{5} V}{15} \frac{k^{4} T^{3}}{h^{3} c^{3}}-\pi V \delta^{2}\left(\frac{32 \pi^{2}}{3}+\frac{44 \pi^{4}}{15}+192 \zeta(3)\right) \frac{k^{4} T^{3}}{h^{3} c^{3}}
$$

At the case of $q=1$, we recover the result for an ordinary Bose gas. To show the effect of deformation on the specific heat, we give a plot of the specific heat per unit volume of a deformed Bose gas vs temperature for different deformation parameters in Fig. 4.

From the above discusses, we can also obtain the Planck's formula for the deformed Bose gas, 


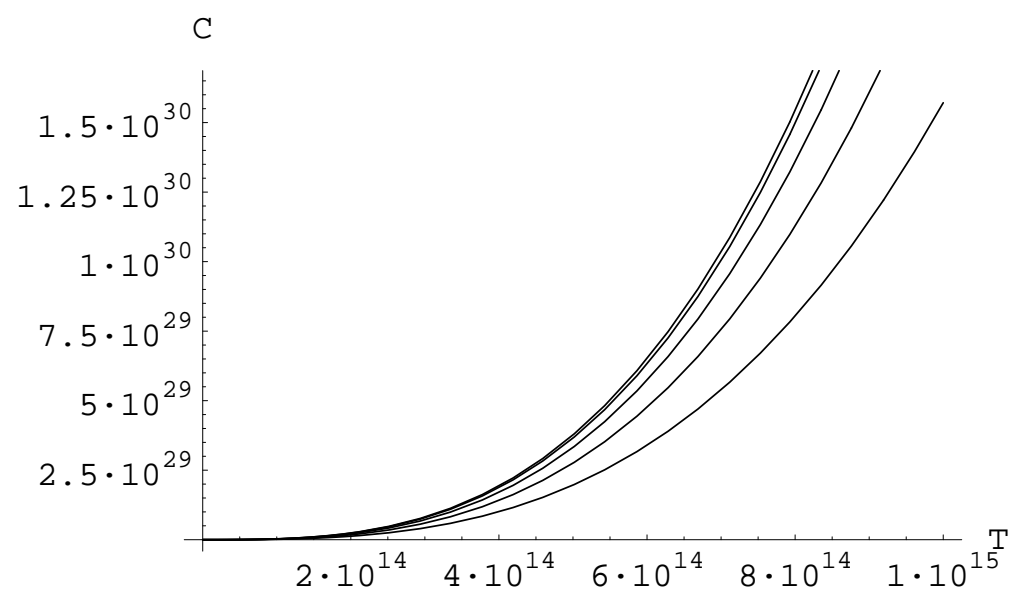

FIG.4 The specific heat per unit volume of a deformed Bose gas

$$
\begin{aligned}
&(\text { start from up } q=1.0,1.1,1.2,1.3,1.4) . \\
& u(\omega, T)= \frac{\hbar \omega^{3}}{c^{3} \pi^{2}\left(e^{\beta \hbar \omega}-1\right)}-\frac{17 \delta^{2} \hbar \omega^{3}}{24 c^{3} \pi^{2}\left(e^{\beta \hbar \omega}-1\right)}-\frac{\delta^{2} \beta \hbar^{2} \omega^{4} e^{\beta \hbar \omega}}{2 c^{3} \pi^{2}\left(e^{\beta \hbar \omega}-1\right)^{2}} \\
&-\frac{\delta^{2} \beta \hbar^{2} \omega^{4} e^{\beta \hbar \omega}}{c^{3} \pi^{2}\left(e^{\beta \hbar \omega}-1\right)^{3}}-\frac{\delta^{2} \beta \hbar^{2} \omega^{4} e^{\beta \hbar \omega}}{2 c^{3} \pi^{2}\left(e^{\beta \hbar \omega}-1\right)^{4}} .
\end{aligned}
$$

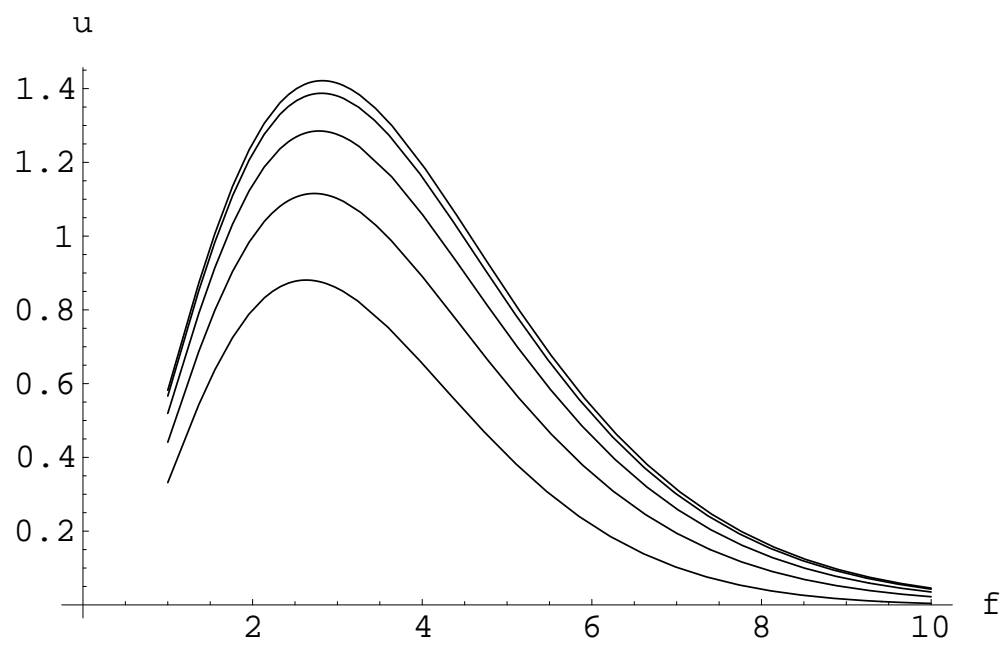

FIG.5 The Planck's

formula of a deformed Bose gas

( start from up $q=1.0,1.1,1.2,1.3,1.4$, here we have used the notation $f=\beta \hbar \omega$.)

In Fig.5, we give a plot of the Planck's formula for the deformed Bose gas. The first term in equation(46) describes an ideal gas consisting of photons. The linearity of the equations of electrodynamics on commutative spacetime guarantees that photons do not interact with one another. However, the other terms in this equation denote 
interactions among photons or nonlinearity of equations of electrodynamics caused by noncommutative deformation of spacetime. At high frequencies $(\beta \hbar \omega \gg 1)$, we have

$$
\begin{aligned}
u(\omega, T)= & \frac{\hbar \omega^{3} e^{-\beta \hbar \omega}}{c^{3} \pi^{2}}-\frac{17 \delta^{2} \hbar \omega^{3} e^{-\beta \hbar \omega}}{24 c^{3} \pi^{2}}-\frac{\delta^{2} \beta \hbar^{2} \omega^{4} e^{-\beta \hbar \omega}}{2 c^{3} \pi^{2}} \\
& -\frac{\delta^{2} \beta \hbar^{2} \omega^{4} e^{-2 \beta \hbar \omega}}{c^{3} \pi^{2}}-\frac{\delta^{2} \beta \hbar^{2} \omega^{4} e^{-3 \beta \hbar \omega}}{2 c^{3} \pi^{2}} .
\end{aligned}
$$

This is the Wien's formula of the deformed Bose gas. We give a plot of the Wien's formula for different deformation parameters in Fig.6. In spite of a complex form of the Planck's formula and the Wien's formula, we note that the density of the spectral frequency distribution of the energy of the deformed Bose gas has a maximum at a frequency $\omega_{m}\left(\beta \hbar \omega_{m} \approx 2.8\right)$, which is roughly independent of deformation parameter. Thus the displacement law is still remained for the deformed Bose gas.

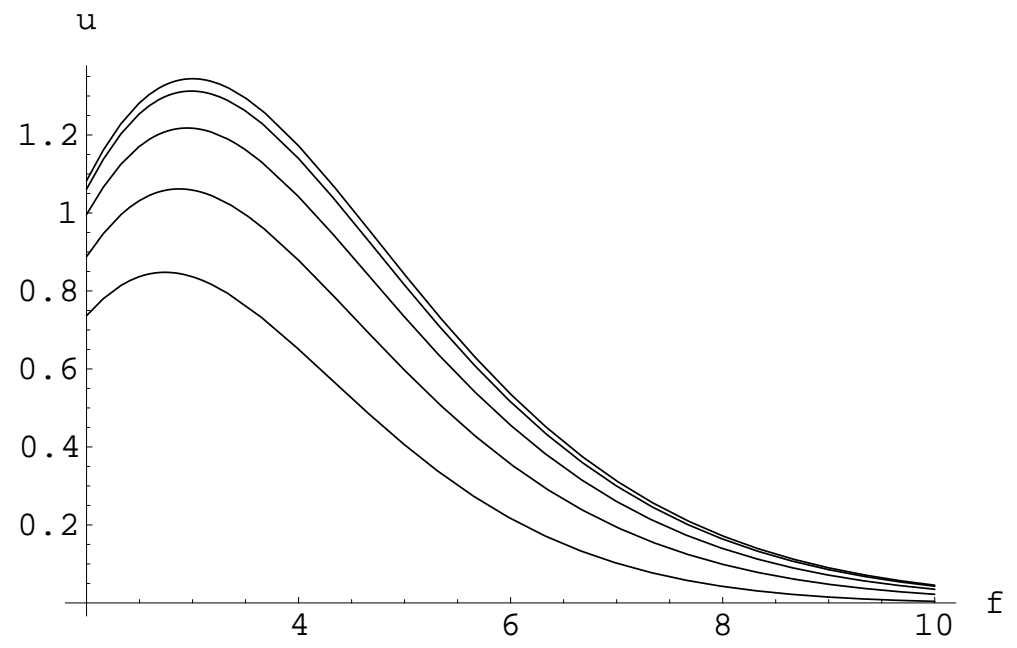

FIG.6 The Wien's formula of a deformed Bose gas ( start from up $q=1.0,1.1,1.2,1.3,1.4$, here we have used the notation $f=\beta \hbar \omega$ ).

In the opposite limiting case of low frequencies $(\beta \hbar \omega \ll 1)$, we get the Rayleigh-Jeans formula for the deformed Bose gas,

$$
\begin{aligned}
u(\omega, T)= & \frac{k T \omega^{2}}{c^{3} \pi^{2}}-\frac{17 \delta^{2} k T \omega^{2}}{24 c^{3} \pi^{2}}-\frac{\delta^{2} k T \omega^{2}}{2 c^{3} \pi^{2}}\left(\frac{\hbar \omega}{k T}+1\right) \\
& -\frac{\delta^{2}(k T)^{2} \omega}{c^{3} \pi^{2} \hbar}\left(\frac{\hbar \omega}{k T}+1\right)-\frac{\delta^{2}(k T)^{3}}{2 c^{3} \pi^{2} \hbar^{2}}\left(\frac{\hbar \omega}{k T}+1\right) .
\end{aligned}
$$


Fig.7 shows a graph corresponding to the Rayleigh-Jeans formula with different deformation parameters.

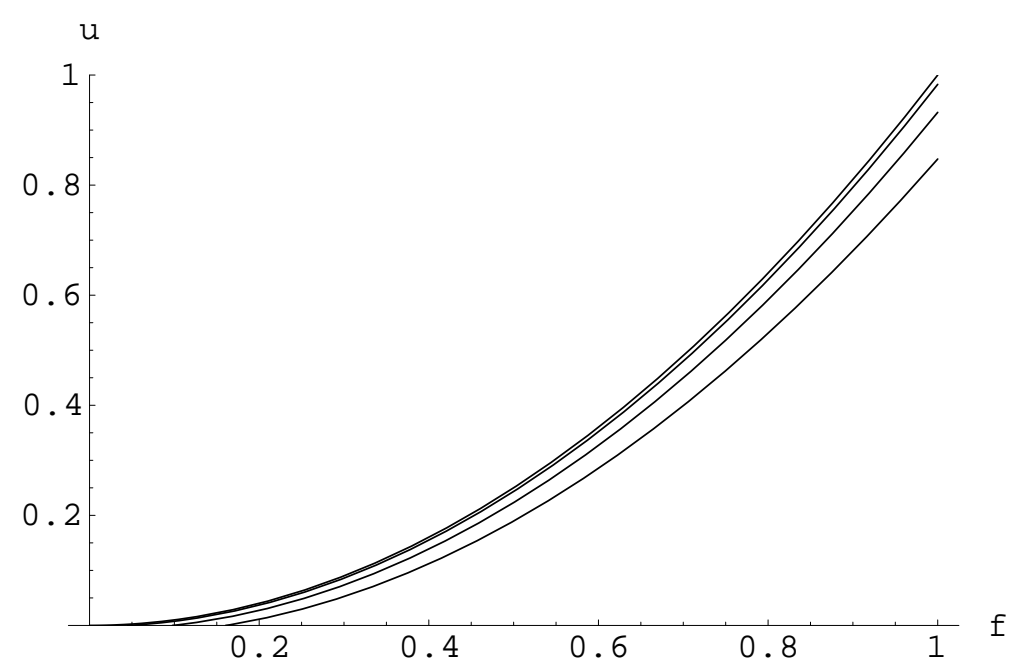

FIG.7 The Rayleigh-Jeans formula of a deformed Bose gas ( start from up $q=1.00,1.06,1.12,1.18$ ).

We obtain the Stefan-Boltzmann's law for the deformed Bose gas of the form,

$$
J=\frac{\pi^{2} k^{4}}{60 \hbar^{3} c^{2}} T^{4}-\frac{\delta^{2} k^{4}}{32 \pi^{2} \hbar^{3} c^{2}}\left(\frac{8 \pi^{2}}{3}+\frac{11 \pi^{4}}{15}+48 \zeta(3)\right) T^{4} .
$$

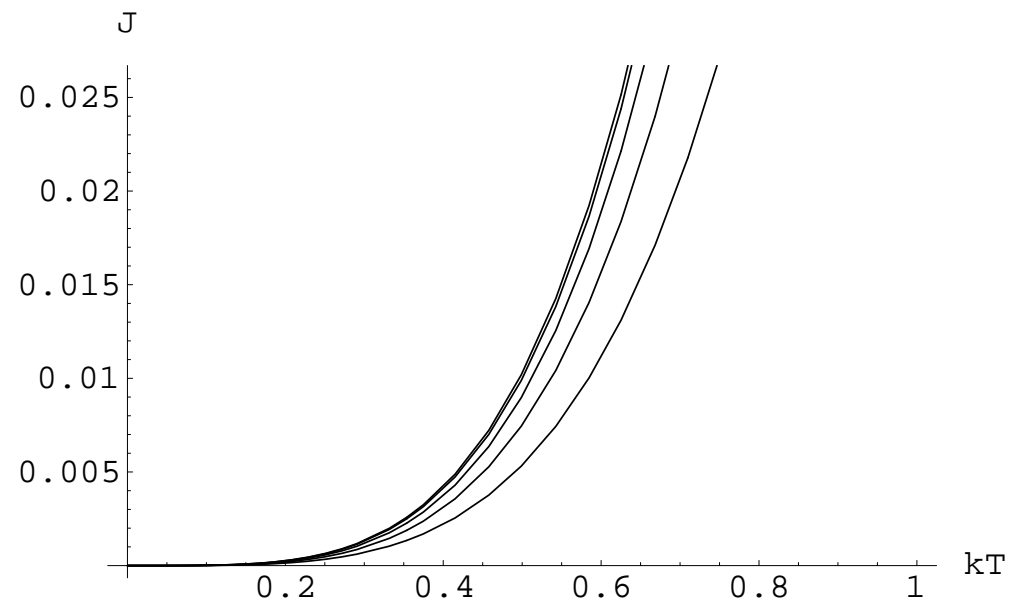

FIG.8 The Stefan-Boltzmann law of a deformed Bose gas ( start from up $q=1.0,1.1,1.2,1.3,1.4$ ).

Similar to the case of an ideal Bose gas, the total energy of the deformed Bose gas is proportional to the fourth power of the temperature. But the Stefan-Boltzmann's 
constant is effectively reduced by the noncommutative deformation of geometry. The fact gives also a signal that the deformed Bose gas is a strongly correlated system. We give a plot of the Stefan-Boltzmann's law for different deformation parameters in Fig.8.

In an adiabatic expansion (or compression) process, the entropy of the system is a constant and the valume and temperature are related by $V T^{3}=$ constant. From (42), we can get the equation of state,

$$
P V^{4 / 3}=c_{q}
$$

\section{Concluding Remarks}

The noncommutative geometry plays a very important role in the study of the Planck scale physics. Quantum field theory and superstring theory (or M theory) on noncommutative spacetime have been studied for a long time. It is well-known that the most realistic laboratory for testing the Planck scale physics is the early universe. The very early universe was opaque due to constant interchange of energy between matter and radiation. It is clear that the radiation field at the early universe can not be described well by an ideal Bose gas system. There should be strong correlations among photons at this stage of evolution of the universe. In fact, attention has been paid recently to the investigation on the deformed radiation dominated universe. Preliminary results show that spacetime quantization gives rise to inflation as the radiation temperature exceeds the Planck temperature. Of course, the study in this direction is only on its very beginning. Many efforts should be made to set up a self-contained inflation theory on noncommutative spacetime. We still have little knowledge on thermodynamics of the deformed radiation field.

From the linearity of the equations of electrodynamics on commutative spacetime, we know that photons do not interact with one another. The thermodynamics of the photonic field can be described well by an ideal Bose gas system. However, the

photons in the deformed radiation fields have strong correlations among themselves. 
The strong correlations among the photons are caused by the nonlinearity of equations of electrodynamics on noncommutative spacetime. It is a difficult task to deal with the interactions among the photons. Numerous papers have been published[33]-[46] on the thermodynamics of the deformed photonic field. However, we still have not got a satisfactory distribution function of the deformed Bose gas.

In this paper, we have treated the deformed photonic field as a strongly correlated system by making use of the Green's function theory. Equations of motion of the Green's function for the deformed photonic field was set up. The Tyablikov procedure[57] was used to decouple these equations of motion. A general form of statistical distribution function for the deformed photonic field was got systematically. Based on this distribution function, we calculated the free energy, the entropy and the specific heat of the deformed photonic field in detail. The equation of state was obtained. We found that the Planck's formula for the deformed Bose gas is really different from the ordinary one. We got terms in Planck's formula, which correspond to the interactions among photons. Similar to the case of an ideal Bose gas, the total energy of the deformed Bose gas is proportional to the fourth power of the temperature. But the Stefan-Boltzmann's constant is effectively reduced by the noncommutative deformation of geometry. We noted that the density of the spectral frequency distribution of the energy of the deformed Bose gas has a maximum at a frequency $\omega_{m}\left(\beta \hbar \omega_{m} \approx 2.8\right)$, which is roughly independent of deformation parameter. Thus the displacement law is still

remained for the deformed Bose gas. The results obtained in the paper can be used to investigate properties of the early universe (e.g. inflation model on noncommutative spacetime). All of these works are in progress.

\section{Acknowledgements}

The work was supported in part by the Natural Science Foundation of China. 


\section{References}

[1] J. Wess, Int. J. Mod. Phys. A 12, 4997 (1997).

[2] N. Seiberg and E. Witten, JHEP 9909, 032 (1999).

[3] Z. Chang, Phys. Rev. D 61, 044009 (2000).

[4] T. Banks, W. Fischler, S. H. Shenker, and L. Susskind, Phys. Rev. D 55, 5112 (1997)

[5] A. Connes, M. R. Douglas, and A. Schwarz, JHEP 9802, 003 (1998).

[6] A. Connes, Noncommutative Geometry, Academic Press, London (1994).

[7] M. R. Douglas and N. A. Nekrasov, Rev. Mod. Phys. 73, 977 (2002).

[8] A. H. Guth, Phys. Rev. D23, 347 (1981).

[9] S. Hancock et al., Nature 367, 333 (1994).

[10] C. H. Lineweaver et al., Ap. J. 448, 482 (1995).

[11] A. Linde, D. Linde, and A. Mezhlumian, Phys. Rev. D49, 1783 (1994).

[12] A. Linde, "Lectures on inflationary cosmology", hep-th/9410082.

[13] J. Martin and R. H. Brandenberger, Phys. Rev. D63, 123501 (2001).

[14] J. C. Niemeyer, Phys. Rev. D63, 123502 (2001).

[15] J. Kowalski-Glikman, Phys. Lett. B499, 1 (2001).

[16] J. C. Niemeyer and R. Parentani, Phys. Rev. D64, 101301 (2001).

[17] R. H. Brandenberger and J. Martin, Mod. Phys. Lett A16, 999 (2001).

[18] J. A. Gu, P. M. Ho, and S. Ramgoolam, hep-th/0101058. 
[19] S. Alexander and J. Magueijo, hep-th/0104093.

[20] A. Kempf, Phys. Rev. D63, 083514 (2001).

[21] C. S. Chu, B. R. Greene, and G. Shiu, Mod. Phys. Lett. A16, 2231 (2001).

[22] A. Kempf and J. C. Niemeyer, Phys. Rev. D64, 103501 (2001).

[23] R. Easther, B. R. Greene, W. H. Kinney, and G. Shiu, Phys. Rev. D64, 103502 (2001).

[24] S. Alexander, R. Brandenberger, and J. Magueijo, hep-th/0108190.

[25] M. Chaichian, D. Ellinas, and P. Kulish, Phys. Rev. Lett. 65, 980 (1990).

[26] Z. Chang, Phys. Rev. A46, 5865 (1992).

[27] Z. Chang, Phys. Rev. A47, 5017 (1993).

[28] E. Celeghini, M. Rasetti, and G. Vitiello, Phys. Rev. Lett. 66, 2056 (1991).

[29] Z. Chang, Phys. Rev. A46, 5860 (1992).

[30] Z. Chang, H. Y. Guo, and H. Yan, Phys. Lett. A156, 192 (1991).

[31] Z. Chang and H. Yan, Phys. Rev. A43, 6043 (1991).

[32] Z. Chang, Phys. Rept. 262, 137 (1995).

[33] P. Angelopoulou et al., J. Phys. A: Math. Gen. 27, L605 (1994).

[34] M. A. Martín-Delgado, J. Phys. A: Math. Gen. 24, L1285 (1991).

[35] S. Vokos and C. Zachos, Mod. Phys. Lett. A9, 1 (1994).

[36] J. A. Tuszyński, J. L. Rubin, J. Meyer, and M. Kibler, Phys. Lett. A175, 173 (1993). 
[37] M. L. Ge and G. Su, J. Phys. A: Math. Gen. 24, L721 (1991).

[38] J. L. Birman, Phys. Lett. A167, 363 (1992).

[39] Y. J. Ng, J. Phys. A: Math. Gen. 23, 1023 (1990).

[40] J. W. Goodison and D. J. Toms, Phys. Lett. A195, 38 (1994); 198, 471 (1995).

[41] R. A. Campos, Phys. Lett. A184, 173 (1994).

[42] S. L. Dalton and A. Inomata, Phys. Lett. A199, 315 (1995).

[43] G. Su and M. L. Ge, Phys. Lett. A173, 17 (1993).

[44] D. I. Fivel, Phys. Rev. Lett. 65, 3361 (1990).

[45] A. Inomata, Phys. Rev. A52, 932 (1995).

[46] M. R. Ubriaco, Phys. Rev. E55, 291 (1997).

[47] A. J. Macfarlane, J. Phys. A: Math. Gen. 22, 4581 (1989).

[48] L. C. Biedenharn, J. Phys. A: Math. Gen. 22, L873 (1989).

[49] C. P. Sun and H. C. Fu, J. Phys. A: Math. Gen. 22, L983 (1989).

[50] X. C. Song, J. Phys. A: Math. Gen. 23, L821 (1990).

[51] Z. Chang, W. Chen, and H. Y. Guo, J. Phys. A: Math. Gen. 23, 4185 (1990).

[52] Z. Chang, W. Chen, H. Y. Guo, and H. Yan, J. Phys. A: Math. Gen. 23, 5371 (1990).

[53] Z. Chang, W. Chen, H. Y. Guo, and H. Yan, J. Phys. A: Math. Gen. 24, 1427 (1991).

[54] Z. Chang, S. M. Fei, H. Y. Guo, and H. Yan, J. Phys. A: Math. Gen. 24, 5435 (1991). 
[55] D. N. Zubarev, Sov. Phys. -Usp. 3, 320 (1960).

[56] Z. Chang, Ann. Phys. (N.Y.) 271, 233 (1999).

[57] S. V. Tyablikov, Ukrain. Mat. Zh. 11, 287 (1959). 